\title{
Identifying robustness in the regulation of collective foraging of ant colonies using an interaction-based model with backward bifurcation
}

\author{
Oyita Udiani $^{1}$, Noa Pinter-Wollman ${ }^{2}$ and Yun Kang ${ }^{3}$
}

\begin{abstract}
Collective behaviors in social insect societies often emerge from simple local rules. However, little is known about how these behaviors are dynamically regulated in response to environmental changes. Here, we use a compartmental modeling approach to identify factors that allow harvester ant colonies to regulate collective foraging activity in response to their environment. We propose a set of differential equations describing the dynamics of: (1) available foragers inside the nest, (2) active foragers outside the nest, and (3) successful returning foragers, to understand how colony-specific parameters, such as baseline number of foragers, interactions among foragers, food discovery rates, successful forager return rates, and foraging duration might influence collective foraging dynamics, while maintaining functional robustness to perturbations. Our analysis indicates that the model can undergo a forward (transcritical) bifurcation or a backward bifurcation depending on colony-specific parameters. In the former case, foraging activity persists when the average number of recruits per successful returning forager is larger than one. In the latter case, the backward bifurcation creates a region of bistability in which the size and fate of foraging activity depends on the distribution of the foraging workforce among the model's compartments. We validate the model with experimental data from harvester ants (Pogonomyrmex barbatus) and perform sensitivity analysis. Our model provides insights on how simple, local interactions can achieve an emergent and robust regulatory system of collective foraging activity in ant colonies.
\end{abstract}

Keywords: Foraging Dynamics; Transcritical Bifurcation; Backward Bifurcation; Bi-stability; Robustness

\section{Introduction}

The regulation of collective foraging by social insect colonies emerges from local rules followed by individual workers [1]. Because social insects live in a dynamic environment, it is important to understand how these individual-based local rules allow the colony to respond to perturbations inside and outside the nest $[2,3,4]$. Collective foraging of social insects has been extensively studied in many systems, revealing similarities in the dynamics of its regulation across species (e.g., bees[5, 6]; ants, [4, 7], etc). For example, ant species exhibit a variety of foraging strategies that employ a combination of direct and indirect social cues [8]. Forager recruitment strategies range from leader-based recruitment in which successful foragers guide recruits directly to the food source (i.e., tandem running; $[9,10]$ ) to self-organizing pheromone trail networks that allow naive recruits to locate and exploit profitable food sources [11].

Mathematical models have been instrumental in uncovering fundamental mechanisms that underlie the foraging dynamics of ant colonies in various environmental conditions [6,12, 13, 14]. Although several models have addressed the dynamics of resource exploitation using pheromone trails (e.g., $[6,12,14,15])$ few have considered systems without such spatial cues. For example, Dussutour \& Nicolis [16] found that the reliability

\footnotetext{
${ }^{1}$ Simon A. Levin Mathematical, Computational and modeling Sciences Center, Arizona State University, Tempe, AZ 85287, USA(oyita.udiani@asu.edu).

${ }^{2}$ BioCircuits Institute, University of California, San Diego, La Jolla CA. 92093-0328 USA (nmpinter@ucsd.edu).

${ }^{3}$ Sciences and Mathematics Faculty, School of Letters and Sciences, Arizona State University, Mesa, AZ 85212, USA (yun.kang@asu.edu).
} 
of collective foraging decisions in a dynamic resource environment is regulated using both pheromone trails and direct interactions. Their analytical and empirical results suggest that interaction based recruitment provides the colony with greater flexibility than pheromone trails when obtaining ephemeral food sources. Here we examine a system in which interactions are the primary mode of communication in an environment with a relatively stable food source.

Colonies of the seed harvester ant Pogonomyrmex barbatus regulate their foraging activity in response to environmental conditions. Harvester ants obtain most of their water by metabolizing fats stored in the seeds they eat [23]. However, the longer a worker forages in the hot desert sun, the less likely it is to return to the nest with food [23]. Therefore, colonies must constantly balance desiccation costs with the expected benefits of finding food [24]. In addition, depredation of active foragers by horned lizards is another ecological pressure that colonies face [35]. Previous studies suggest that the rate at which foraging activity resumes after a predator attack is related to the duration of the predation event $[4,17]$.

Foraging regulation is mediated by brief interactions among workers $[4,17,18,19,20]$. Foragers leave the nest in search of seeds after reaching a threshold number of antennal interactions with successful returning foragers in the area just inside the nest entrance, called the vestibule [17, 21]. Because foragers continue their search for food until a seed item is found, forager return rates may serve as a reliable proxy for external conditions such as seed availability, humidity, etc. [22]. Antennal contacts inside the nest are used to sense cuticular hydrocarbons which provide information about an ant's task [19, 25, 26]. Furthermore, when encountering the combined odor of foragers and seeds at a particular rate, an inactive forager will leave the nest and look for food [17, 21]. Thus, interactions among workers in the vestibule are fundamental for regulating foraging activity in response to environmental perturbations. Indeed, recent studies by PinterWollman et al. [17] and Gordon et al. [18] demonstrate that colonies reliably adjust their foraging intensity relative to changes in forager return rates over the course of just a few minutes.

To date, only a few models have attempted to investigate the effect of physical interactions on the dynamics of collective activity [16, 27, 28]. A notable contribution along these lines was a recent work by Prabhakar et al. [29], which explored the regulation of foraging activity in P. barbatus using a simple stochastic algorithm. Approximating the inter-arrival times of successive returning foragers as a Poisson process, they generated a simple linear relation between the rates of returning foragers and outgoing foragers by assuming that each returning forager increased the rate of outgoing foragers by a fixed amount. Although their model captured many aspects of the data, including previously reported correlations between numbers of returning and outgoing foragers during periods of high food availability [17, 30,31], the authors conceded some limitations. In short, their model could neither account for the non-linear patterns of forager interaction, nor mechanistically define parameters, which influence the rate of returning foragers and their effects on inactive foragers in the nest.

In this paper, we develop a simple compartmental model based on the foraging behavior of harvester ant colonies. Our model captures the mechanistic regulation of collective foraging activity by defining the interactions among three categories of foraging ants: (i) available foragers at the nest, (ii) active foragers leaving the nest, and (iii) successful returning foragers [17]. We then analyze the sensitivity of the model to changes in its parameters to understand how colony-specific properties, such as interaction rates among workers, and environmental parameters, such as food availability, can influence the robustness of foraging activity. Furthermore, we validate our model by comparing its dynamics under simulated perturbations to empirical observations of a forager removal experiment described in [17].

The rest of the paper is structured as follows: In section 2, we develop the foraging model with general parameters. In section 3, we summarize the complete mathematical analysis of its dynamics and provide relevant biological implications. In section 4 , we validate the model by comparing the results of simulated perturbations with the empirical findings of [17], and present results on the sensitivity analysis. In section 5, we discuss our results and include some closing remarks. The detailed proofs of our main analytical results are presented in section 6 . 


\section{Model Derivation}

We develop a deterministic system of nonlinear differential equations to describe the regulation of foraging activity in harvester ant colonies (Fig. 1). The model's state variables are defined as follows: let $N(t)=$ $A(t)+F(t)+R(t)$ be the total forager workforce of a focal colony at time $t$; where: $A(t)$ denotes the number of available foragers inside the nest's vestibule, $F(t)$ denotes the number of outgoing (active) foragers, and $R(t)$ denotes the number of returning foragers. The model's structure follows from the state-based framework outlined by Sumpter \& Pratt [13] along with some formalistic assumptions. Most importantly, we assume that the total workforce is large to avoid the effects of stochasticity in small populations. In light of this caveat, we focus our model on the minute to minute dynamics of a mature colony which can have up to several thousand foragers over the course of hourly activity [32, 33]. Furthermore, we make the following assumptions about its state dynamics:

1. Available Foragers $A$ : The numbers of available foragers $A(t)$ is determined by the following four flow rates.

The inflow rate consists of two components:

(a) The arrival rate, $\Lambda(t)=k_{1}$ which describes the movement of potential foragers from the inner nest to the vestibule. We assume that this rate is constant in our model. In nature, $k_{1}$ might vary over the course of the day or throughout the year depending on properties such as the number of workers allocated to the foraging task, colony age, etc. [32, 34].

(b) The forager turnover rate, $\Upsilon(t)=\gamma$ which describes the rate at which returning foragers become re-available for recruitment. This rate will be influenced by the distance between the resource site and the nest, searching and handling times, as well as the total amount of time spent inside the nest after a successful trip (e.g., depositing seeds). We aggregate these effects into a single constant $(1 / \gamma)$, which describes the average time spent as a returning forager, and thus also affects the rate at which foragers are recruited as detailed below.

The outflow rate consists of two components:

(a) The activation (recruitment) rate of available foragers, $\Psi(A, R)=\beta A R$ which describes the rate of increase in the number of active foragers. This formulation assumes mass action incidence and is supported by the empirical observations of $[17,19]$. More specifically, we assume that: (i) an available forager contacts only the fraction $(\rho)$ of returning foragers that are inside the vestibule at an average rate of $\mathrm{c}$ interactions per unit time, (ii) interactions are independent of one another and are equally likely to occur with any available forager in the vestibule, and (iii) each interaction has a constant probability $(\mu)$ of activation. Hence, the "effective interaction rate" $(\beta)$ is the product of the number of interactions per unit time made by a returning forager that is inside the vestibule and the probability that an interaction activates a new forager: $\beta=c \rho \mu$.

(b) The retirement rate of available foragers from the vestibule into the inner nest, $\chi(A, R)=k_{2} \frac{A}{1+R}$ which reflects the behavior of available foragers in response to changes in forager return rate reported in [17]. When forager return rates are low, (i.e., $R$ is small) the number of available foragers is large relative to the average number of returning foragers inside the vestibule. As such, available foragers are more likely to retire to the inner nest [17]. This occurs at a maximum rate $k_{2} A$ (when there are no returning foragers: $R=0$ ). On the other hand, when forager return rates are high (i.e., $R$ is large) then available foragers are less likely to retire into the inner nest.

Based on these assumptions, the rate equation for the number of available foragers is given by:

$$
A^{\prime}=\underbrace{k_{1}}_{\text {arrive }}+\underbrace{\gamma R}_{\text {turnover }}-\underbrace{\beta A R}_{\text {activate }}-\underbrace{k_{2} \frac{A}{1+R}}_{\text {retire }}
$$


2. Active Foragers $F$ : The number of active foragers $F(t)$ is determined by the recruitment rate $\beta A R$; the rate of resource discovery, $\alpha(S) F$; and the loss (or death) rate $d_{f} F$ while searching for the resource (e.g. due to predatory activity near foraging trails [35]). The rate $\alpha(S) F$ at which active foragers $F$ become returning foragers $R$ is influenced by the abundance of available seed items, $S$. For mature harvester ant colonies, the effective seed densities in the nest's home range are typically orders of magnitude greater than the maximum number of foragers [30]. Thus, seed abundance should be generally (approximately) independent of a colony's foraging intensity especially on the timescale of hourly activity. Hence, we will assume that the per-capita resource discovery rate is constant: $\alpha(S)=\alpha$. We also assume foragers continue to search until they discover a seed item as is typical for harvester ants [22]. Based on these ecological assumptions, the rate equation for the number of active foragers is given by:

$$
F^{\prime}=\underbrace{\beta A R}_{\text {activate }}-\underbrace{\alpha F}_{\text {discover food }}-\underbrace{d_{f} F}_{\text {dead/lost }}
$$

3. Returning foragers $R$ : The number of returning foragers $R(t)$ is determined by the rate at which active foragers discover food items, $\alpha F$; the rate at which foragers return to the nest and become re-available for recruitment $\gamma R$; as well as the rate of predation and/or loss while en route to the nest $d_{r} R$. Based on these assumptions, the rate equation for the number of returning foragers is given by:

$$
R^{\prime}=\underbrace{\alpha F}_{\text {discover food }}-\underbrace{\gamma R}_{\text {turnover }}-\underbrace{d_{r} R}_{\text {dead/lost }}
$$

Thus, the collective dynamics and regulation of foraging in harvester ants may be represented by the following system, hereafter referred to as model (4):

$$
\begin{aligned}
A^{\prime} & =k_{1}-\beta A R-k_{2} \frac{A}{1+R}+\gamma R \\
F^{\prime} & =\beta A R-\left(\alpha+d_{f}\right) F \\
R^{\prime} & =\alpha F-\left(\gamma+d_{r}\right) R
\end{aligned}
$$

\begin{tabular}{c|l|c|c|c}
\hline Parameter & Description & Range & Default & Source \\
\hline \hline$k_{1}$ & Arrival rate $\left(\right.$ ants $\left.\cdot \mathrm{sec}^{-1}\right)$ & $(0,5)$ & 0.5 & {$[17]$} \\
$k_{2}$ & Retirement rate $\left(\right.$ ants $\left.\cdot \mathrm{sec}^{-1}\right)$ & $(0,5)$ & 0.2 & {$[17]$} \\
$\beta$ & Effective interaction rate $\left(\right.$ ants $\left.\cdot \mathrm{sec}^{-1}\right)$ & $(0,2)$ & 0.1 & {$[17]$} \\
$\gamma$ & Forager turnover rate $\left(\mathrm{sec}^{-1}\right)$ & $(0,5)$ & 0.03 & {$[4,17,33]$} \\
$\alpha$ & Resource discovery rate $\left(\mathrm{seeds}^{-1} \mathrm{sec}^{-1}\right)$ & $(0,1)$ & 0.04 & {$[30,36,37]$} \\
$d_{f}$ & Loss rate: outgoing foragers $\left(\mathrm{sec}^{-1}\right)$ & $(0,1)$ & 0.005 & {$[38]$} \\
$d_{r}$ & Loss rate: returning foragers $\left(\mathrm{sec}^{-1}\right)$ & $(0,1)$ & 0.025 & {$[38]$} \\
\hline
\end{tabular}

Table 1: Definition of parameters used in model (4). Sampling ranges for parameter estimation were compiled (or approximated) from cited sources. Default values were used for model simulations, and sensitivity analysis in section 4 . 




Figure 1: Flow diagram of the proposed foraging model (4). The dynamics of the rectangular components (i.e., Inner Nest $\mathbb{E}$ Resource $S$, are assumed to be independent of the colony's foraging dynamics over the timescale of our model (i.e., minutes). Parameters are defined in Table 1.

\section{Mathematical Analysis}

In this section, we give a complete mathematical description of model (4) which includes a bifurcation analysis, that reveals the biological conditions under which the colony can reliably forage at stable levels. We uncover a range of parameters within which our model exhibits bistability (i.e., colonies may either forage or not) and outline how the relationship among the various parameters influences this outcome. We begin by establishing the basic dynamical properties of model (4), including specification of equilibria and their stability conditions (Theorem 3.1-3.2). We then summarize its global behavior in terms of possible bifurcations (Corollary 3.1), and discuss their relevant biological implications.

Theorem 3.1. [Compact Attractor] The foraging model (4) is positively invariant in $\mathbb{R}_{+}^{3}$ and every trajectory starting in $\mathbb{R}_{+}^{3}$ is attracted to the following compact set $C$ :

$$
C=\left\{(A, F, R) \in \mathbb{R}_{+}^{3}: \frac{k_{1}}{\max \left\{k_{2}, d_{f}, d_{r}\right\}} \leq N \leq \frac{k_{1}+\frac{k_{1}^{2}}{4 d_{r}}}{\min \left\{k_{2}, d_{f}, d_{r}\right\}}\right\}
$$

where $N=A+F+R$.

Notes: Theorem 3.1 implies that model (4) is well-defined (i.e., biologically plausible) because the total number of foragers $(N)$ is bounded as observed in nature [33], and all compartments remain nonnegative for nonnegative initial conditions. In addition, the number of available foragers, A, is always non-zero if the worker arrival rate $\left(k_{1}\right)$ is positive. See section 6 for a detailed proof. 


\subsection{Equilibria and their stability}

An equilibrium of model (4) should satisfy the following equations:

$$
\begin{aligned}
& R^{\prime}=\alpha F-\left(\gamma+d_{r}\right) R=0 \Leftrightarrow F=\frac{\gamma+d_{r}}{\alpha} R \\
& F^{\prime}=\beta A R-\left(\alpha+d_{f}\right) F=0 \Leftrightarrow A=\frac{\left(\alpha+d_{f}\right) F}{\beta R}=\frac{\left(\alpha+d_{f}\right) \frac{\gamma+d_{r}}{\alpha R} R}{\beta R}=\frac{\left(\alpha+d_{f}\right)\left(\gamma+d_{r}\right)}{\alpha \beta} \\
& N^{\prime}=k_{1}-k_{2} \frac{A}{1+R}-d_{f} F-d_{r} R=0 \Leftrightarrow k_{1}-k_{2} \frac{A}{1+R}-\frac{d_{f}\left(\gamma+d_{r}\right)}{\alpha} R-d_{r} R=0 .
\end{aligned}
$$

where $N^{\prime}=A^{\prime}+F^{\prime}+R^{\prime}$. Thus, we have the following two cases:

1. When $F=R=0$, model (4) has a unique non-foraging equilibrium: $\mathscr{E}_{0}=\left(A_{0}^{*}, 0,0\right)=\left(\frac{k_{1}}{k_{2}}, 0,0\right)$. This equilibrium always exists. Note that $A_{0}^{*}=\frac{k_{1}}{k_{2}}$ defines a baseline number of available foragers in the vestibule in the absence of returning foragers.

2. When $F \neq 0, R \neq 0$, model (4) has at most two foraging equilibria: $\mathscr{E}_{i}=\left(A_{f}^{*}, F_{i}^{*}, R_{i}^{*}\right)=\left(A_{f}^{*}, \frac{\gamma+d_{r}}{\alpha} R_{i}^{*}, R_{i}^{*}\right)$ $i=1,2$ where;

$$
A_{f}^{*}=\frac{\left(\alpha+d_{f}\right)\left(\gamma+d_{r}\right)}{\alpha \beta}>\frac{\gamma}{\beta} \Rightarrow \beta A_{f}^{*}-\gamma>0
$$

and $R_{i}^{*}$ are the roots of the equation:

$$
\phi\left(R^{*}\right)=\left[k_{1}-\left(\beta A_{f}^{*}-\gamma\right) R^{*}\right]\left(1+R^{*}\right)=k_{2} A_{f}^{*} .
$$

To see this, note that an interior foraging equilibrium necessarily satisfies:

$$
N^{\prime}=A^{\prime}+F^{\prime}+R^{\prime}=k_{1}-k_{2} \frac{A_{f}^{*}}{1+R^{*}}-\frac{d_{f}\left(\gamma+d_{r}\right)}{\alpha} R^{*}-d_{r} R^{*}=\frac{\left[k_{1}-\left(\beta A_{f}^{*}-\gamma\right) R\right](1+R)-k_{2} A_{f}^{*}}{1+R^{*}}=\frac{\phi\left(R^{*}\right)-k_{2} A_{f}^{*}}{1+R^{*}}=0
$$

The explicit forms of $R_{i}^{*}$ are solved from the equation $\phi\left(R^{*}\right)=k_{2} A_{f}^{*}$ :

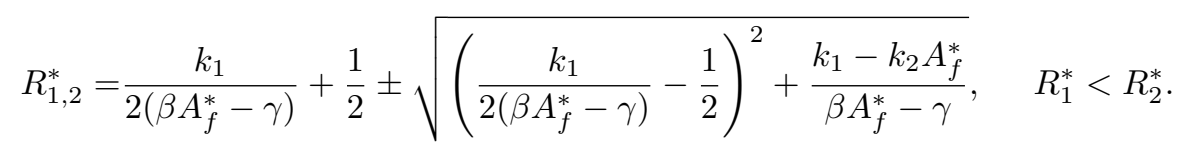

To continue our analysis, we make the following observations and define quantities which largely determine the dynamics of Model (4):

1. First, notice that the equation $\phi(R)=\left[k_{1}-\left(\beta A_{f}^{*}-\gamma\right) R\right](1+R)$ has a unique maximum $\phi_{\max }$ at its critical point:

$$
R_{c}=\frac{\gamma-\beta A_{f}^{*}+k_{1}}{2\left(\beta A_{f}^{*}-\gamma\right)}=\frac{\alpha\left(k_{1}-d_{r}\right)-d_{f}\left(\gamma+d_{r}\right)}{2\left[\alpha d_{r}+d_{f}\left(\gamma+d_{r}\right)\right]} .
$$

Mathematically, $\phi(R)$ relates the net rate of change of non-retiring (hence, available) foragers with the number of returning foragers, which represents the net average rate of forager availability. It accounts for the expected increase in forager availability, due to decreasing retirement rates of available foragers, as the number of returning foragers increases (see equation (1)). Consequently, $\boldsymbol{R}_{\boldsymbol{c}}$ represents the critical number of returning foragers above which the expected reduction in forager retirement rates is insufficient to increase the net average rate of forager availability (Fig. 2(b)). Thus, we define:

$$
\phi_{\max }=\phi\left(R_{c}\right)=\frac{\left(\beta A_{f}^{*}-\gamma+k_{1}\right)^{2}}{4\left(\beta A_{f}^{*}-\gamma\right)}=\frac{\left[\alpha\left(k_{1}-d_{r}\right)-d_{f}\left(\gamma+d_{r}\right)\right]^{2}}{4 \alpha\left[\alpha d_{r}+d_{f}\left(\gamma+d_{r}\right)\right]} \geq \phi(0)=k_{1}
$$


as the maximum possible rate of forager availability.

2. We define $\mathcal{R}_{0}$ as the forager generation number, where:

$$
\mathcal{R}_{0}=\frac{A_{0}^{*}}{A_{f}^{*}}=\frac{k_{1} / k_{2}}{\frac{\left(\alpha+d_{f}\right)\left(\gamma+d_{r}\right)}{\alpha \beta}}=\frac{k_{1}}{k_{2}} \frac{\alpha \beta}{\left(\alpha+d_{f}\right)\left(\gamma+d_{r}\right)} .
$$

$\mathcal{R}_{0}$ represents the average number of recruits generated by a single returning forager when foraging activity is near the non-foraging equilibrium state. It is the product of the expected production rates of active foragers (via recruitment) and returning foragers (via resource discovery) over the typical duration of a foraging trip:

$$
\mathcal{R}_{0}=\frac{A_{0}^{*}}{A_{f}^{*}}=\underbrace{\beta \frac{k_{1}}{k_{2}}}_{\text {Baseline recruitment rate }} \cdot \underbrace{\alpha}_{\text {Resource discovery rate }} \cdot \underbrace{\frac{1}{\left(\alpha+d_{f}\right)\left(\gamma+d_{r}\right)}}_{\text {Mean foraging duration }}
$$

Thus, $\mathcal{R}_{0}$ is dimensionless, confirming its interpretation as a generation number. Intuitively, foraging activity should persist at the foraging equilibrium state if every returning forager recruits more than one new forager (i.e., $\mathcal{R}_{0}>1$ ) (Fig. 3). However, we note that $\mathcal{R}_{0}$ is ultimately bounded above (i.e., $\left.\mathcal{R}_{0}<\frac{\beta k_{1}}{k_{2}\left(\gamma+d_{r}\right)}\right)$. The biological interpretation of this condition is that the expected return rate of newly activated foragers is always less than the recruitment rate, which simply reflects the fact that it takes time for active foragers to discover food items and return to the nest in our model (Fig. 1).

3. The sign of $\boldsymbol{R}_{\boldsymbol{c}}$ (10) determines the maximum number of interior foraging equilibria. This depends in part on the rate ratio:

$$
\mathcal{R}_{A}=\frac{\beta A_{0}^{*}}{k_{1}+\gamma} .
$$

$\mathcal{R}_{A}$ represents the relative change in forager availability due to a single returning forager near the non-foraging equilibrium state. If $R_{c}<0: \Longrightarrow \alpha k_{1}<\alpha d_{r}+d_{f}\left(\gamma+d_{r}\right) \Leftrightarrow\left(\frac{k_{1}+\gamma}{\beta A_{0}^{*}}\right) \mathcal{R}_{0}=\frac{\mathcal{R}_{0}}{\mathcal{R}_{A}}<1$, then the forager availability rate always decreases for any number of returning foragers. This is because the baseline recruitment rate is always larger than the net production of available foragers over the average foraging duration (i.e., $\left.\beta A_{0}^{*}>\left(k_{1}+\gamma\right) \mathcal{R}_{0}\right)$. In this case, model (4) may have either zero or one interior equilibrium $\mathscr{E}_{2}$ (see Fig. 2(a)). Conversely, if $R_{c}>0: \Longrightarrow \alpha k_{1}>\alpha d_{r}+d_{f}\left(\gamma+d_{r}\right) \Leftrightarrow \frac{\mathcal{R}_{0}}{\mathcal{R}_{A}}>1$, then the forager availability rate may increase for a range of returning foragers. In this case, model (4) may have zero, one $\mathscr{E}_{2}$, or two interior equilibria $\mathscr{E}_{i}, i=1,2$ (see Fig. 2(b)).

4. Model (4) can undergo a backward bifurcation as our analysis reveals later in this section. This bifurcation is characterized by the emergence of a second threshold $\tilde{\mathcal{R}}_{\Delta}$ defined as follows:

$$
\tilde{\mathcal{R}}_{\Delta}=\max \left(\mathcal{R}_{A}, \mathcal{R}_{\Delta}\right)=\frac{\mathcal{R}_{A}+\mathcal{R}_{\Delta}}{2}+\frac{\left|\mathcal{R}_{A}-\mathcal{R}_{\Delta}\right|}{2}
$$

where

$$
\mathcal{R}_{\Delta}=\frac{\phi(0)}{\phi_{\max }}=\frac{k_{1}}{\phi_{\max }}=\frac{4 k_{1}\left(\beta A_{f}^{*}-\gamma\right)}{\left(\beta A_{f}^{*}-\gamma+k_{1}\right)^{2}}=\frac{4 k_{1} \alpha\left[\alpha d_{r}+d_{f}\left(\gamma+d_{r}\right)\right]}{\left[\alpha\left(k_{1}-d_{r}\right)-d_{f}\left(\gamma+d_{r}\right)\right]^{2}} \leq 1
$$

represents the relative contribution of the worker arrival rate $\left(k_{1}\right)$ to the maximum possible rate of forager availability. As discussed in section $6, \tilde{\mathcal{R}}_{\Delta}$ represents a sub-critical threshold where Model (4) goes from having zero to two interior equilibria (Fig. 3(b)). Biologically, however, it defines a lower bound for $\mathcal{R}_{0}$ (i.e., the minimum number of new forager recruits, per returning forager, that are necessary for maintaining foraging activity). 


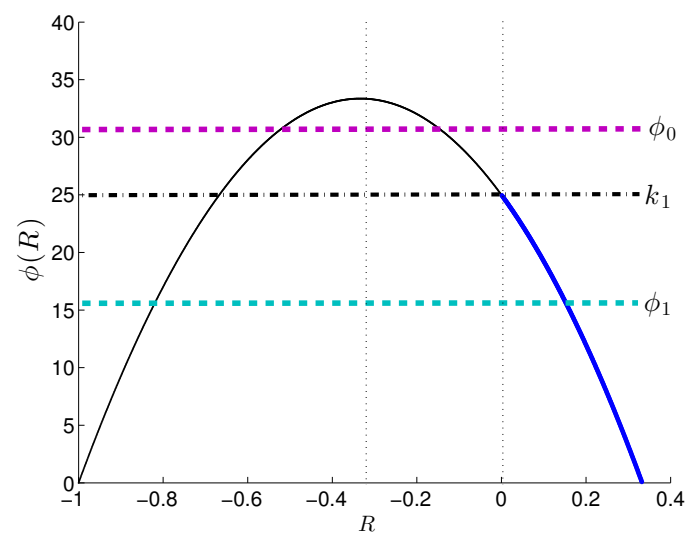

(a) $R_{c}<0$ (i.e., $\left.\frac{\mathcal{R}_{0}}{\mathcal{R}_{A}}<1\right)$ : only $\mathscr{E}_{0}$ or $\left(\mathscr{E}_{0} \& \mathscr{E}_{2}\right)$

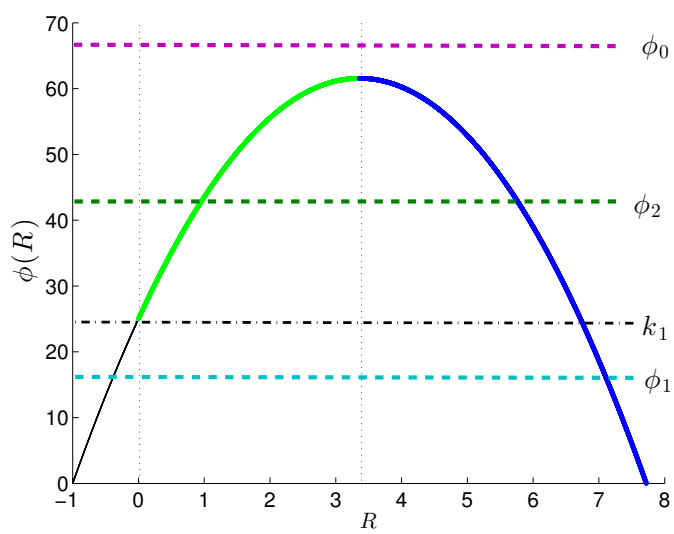

(b) $R_{c}>0$ (i.e., $\left.\frac{\mathcal{R}_{0}}{\mathcal{R}_{A}}>1\right)$ : only $\mathscr{E}_{0}$ or $\left(\mathscr{E}_{i}, i=0,2\right)$ or $\left(\mathscr{E}_{i}, i=\right.$ $0,1,2)$

Figure 2: Schematic nullclines of model (4). Biologically feasible (i.e positive) equilibria occur at intersections between $\phi(R)$ and the dashed horizontal lines $\left(\phi_{i}=k_{2} A_{f}^{*}, i=1,2\right)$ where $\phi_{i}<k_{1}$. (a) If $R_{c}<0$, model (4) may have a unique foraging equilibrium ( $\left.\phi_{1}\right)$. (b) If $R_{c}>0$, model (4) may have a pair of foraging equilibria $\left(\phi_{1,2}\right)$. Equilibria are classified as attractors (blue), saddles (green) or repellers (black). There are no positive equilibria on the line $\phi_{0}$.

The stability of an equilibrium $E^{*}=\left(A^{*}, F^{*}, R^{*}\right)$ is determined by the Jacobian matrix (17) of Model (4) evaluated at the equilibrium:

$$
\left.J\right|_{E^{*}=\left(A^{*}, F^{*}, R^{*}\right)}:=\left[\begin{array}{ccc}
-\beta R^{*}-\frac{k_{2}}{1+R^{*}} & 0 & -\beta A^{*}+\gamma+\frac{k_{2} A^{*}}{\left(1+R^{*}\right)^{2}} \\
\beta R^{*} & -\left(\alpha+d_{f}\right) & \beta A^{*} \\
0 & \alpha & -\left(\gamma+d_{r}\right)
\end{array}\right] .
$$

The following theorem provides the explicit condition on the existence and local stability of the non-foraging equilibrium $\mathscr{E}_{0}$ and the foraging equilibrium $\mathscr{E}_{i}, i=1,2$.

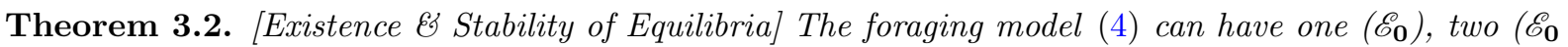
and $\mathscr{E}_{\mathbf{2}}$ ), or three equilibria ( $\mathscr{E}_{\mathbf{0}}$ and $\mathscr{E}_{\mathbf{i}}, i=1,2$ ) depending on the values of $\mathcal{R}_{0}, \mathcal{R}_{A}$, and $\mathcal{R}_{\Delta}$. Sufficient conditions for the existence and local stability of these equilibria are summarized in Table (2).

\begin{tabular}{|c|c|c|}
\hline Equilibria & Existence Condition & Stability Condition \\
\hline $\mathscr{E}_{\mathbf{0}}$ & Always & $\begin{array}{c}\text { Locally stable if } \mathcal{R}_{0}<1 \\
\text { Saddle if } \mathcal{R}_{0}>1\end{array}$ \\
\hline $\mathscr{E}_{\mathbf{1}}$ & $\tilde{\mathcal{R}}_{\Delta}<\mathcal{R}_{0}<1$ & Always a saddle \\
\hline $\mathscr{E}_{\mathbf{2}}$ & $\tilde{\mathcal{R}}_{\Delta}<\mathcal{R}_{0}<1 ;$ or $\mathcal{R}_{0}>1$ & Always locally stable \\
\hline
\end{tabular}

Table 2: Equilibria and stability. $A_{0}^{*}=\frac{k_{1}}{k_{2}}, \mathcal{R}_{0}=\frac{A_{0}^{*}}{A_{f}^{*}}, \mathcal{R}_{A}=\frac{\beta A_{0}^{*}}{k_{1}+\gamma}, \mathcal{R}_{\Delta}=\frac{k_{1}}{\phi_{\max }}$, and $\tilde{\mathcal{R}}_{\Delta}=\max \left(\mathcal{R}_{A}, \mathcal{R}_{\Delta}\right) . A_{f}^{*}$ and $\phi_{\max }$ are given in (7) \& (11) respectively.

Remark 3.1. Theorem 3.2 implies that: (i) $\mathcal{R}_{0}$ determines the stability of the non-foraging equilibrium, (ii) $\mathcal{R}_{0}, \mathcal{R}_{A}$, and $\mathcal{R}_{\Delta}$ determine the existence of the foraging equilibria $\mathscr{E}_{\mathbf{i}}, i=1,2$ (see Table 2 E Fig. 2); and (iii) model (4) only exhibits equilibrium behavior (i.e., there are no periodic or chaotic dynamics). 


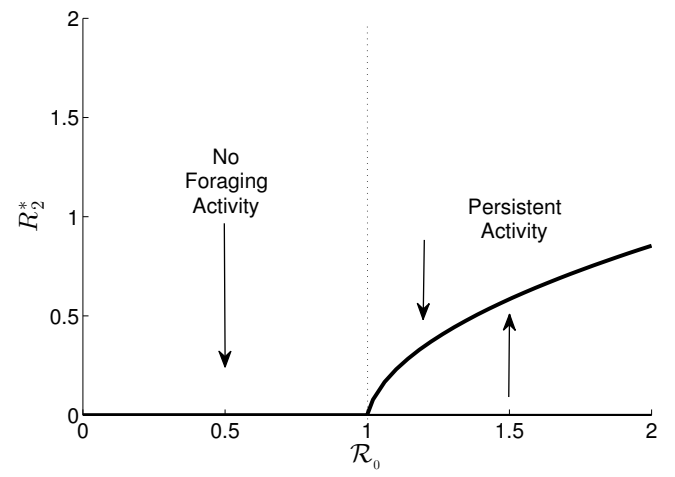

(a) Forward bifurcation when $R_{c}<0$ (i.e., $\frac{\mathcal{R}_{0}}{\mathcal{R}_{A}}<1$ )

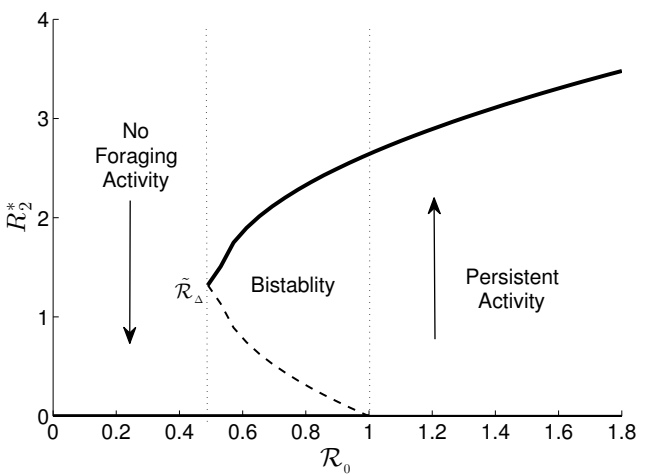

(b) Backward bifurcation when $R_{c}>0$ (i.e., $\frac{\mathcal{R}_{0}}{\mathcal{R}_{A}}>1$ )

Figure 3: One-dimensional bifurcation diagrams for model (4). The origin $\left(R_{2}^{*}=0\right)$ corresponds to the non-foraging equilibrium state which is stable whenever $\mathcal{R}_{0}<1$, and unstable otherwise. (a) A stable interior equilibrium (solid) bifurcates "forward" from the origin when $\mathcal{R}_{0}=1$ (left panel). (b) An unstable interior equilibrium (dashed) bifurcates "backward" from the origin when $\mathcal{R}_{0}=1$ and merges with its stable branch at $\tilde{\mathcal{R}}_{\Delta}$. Note: $\frac{\partial R_{2}^{*}}{\partial \mathcal{R}_{0}}>0$ for $\mathcal{R}_{0}>\tilde{\mathcal{R}}_{\Delta}$.

\subsection{Global dynamics and Bifurcations}

Based on our analytical results shown in Theorem 3.1-3.2, and observations in Remark 3.1 we can classify global dynamics of the foraging model (4) in terms of $\mathcal{R}_{0} \mathcal{R}_{A}$, and $\mathcal{R}_{\Delta}$ as the following theorem:

Corollary 3.1 (Global Dynamics). Depending on the values of $\mathcal{R}_{0} \mathcal{R}_{A}$, and $\mathcal{R}_{\Delta}$, the global dynamics of model (4) can be classified into one of three scenarios (also see Table 3):

1. No activity: If $\mathcal{R}_{0}$ is small such that $\mathcal{R}_{0}<\min \left\{1, \mathcal{R}_{A}\right\}$ or $\mathcal{R}_{0}<\tilde{\mathcal{R}}_{\Delta}<1$, then the foraging model (4) has only the non-foraging equilibrium $\mathscr{E}_{\mathbf{0}}=\left(\frac{k_{1}}{k_{2}}, 0,0\right)$ which is globally stable. Under either of these conditions, the colony will be unable to maintain its foraging activity.

2. Persistent activity: If $\mathcal{R}_{0}$ is large such that $\mathcal{R}_{0}>1$, then the foraging model (4) has the non-foraging equilibrium $\mathscr{E}_{\mathbf{0}}$ and the foraging equilibrium $\mathscr{E}_{\mathbf{2}}$ where $\mathscr{E}_{\mathbf{0}}$ is a saddle and $\mathscr{E}_{\mathbf{2}}$ is globally stable. Under this condition, the colony will persistently reach stable, high levels of activity.

3. Bistablity: If $\mathcal{R}_{0}$ is intermediate in the range $\tilde{\mathcal{R}}_{\Delta}<\mathcal{R}_{0}<1$, then the foraging model (4) has the non-foraging equilibrium $\mathscr{E}_{\mathbf{0}}$ and two foraging equilibria $\mathscr{E}_{\mathbf{i}}, i=1,2$ where both $\mathscr{E}_{\mathbf{0}}$ and $\mathscr{E}_{\mathbf{2}}$ are locally asymptotically stable. $\mathscr{E}_{\mathbf{1}}$ is always a saddle point. Under this condition, the colony can potentially reach intermediate levels of activity depending on initial conditions.

\begin{tabular}{|c|c|c|}
\hline Scenario & Condition & Dynamics \\
\hline Only $\mathscr{E}_{\mathbf{0}}$ & $\begin{array}{c}\mathcal{R}_{0}<\min \left\{1, \mathcal{R}_{A}\right\} \text { or } \\
\mathcal{R}_{0}<\mathcal{R}_{\Delta}<1\end{array}$ & Globally stable. \\
\hline $\mathscr{E}_{\mathbf{0}}$ and $\mathscr{E}_{\mathbf{2}}$ & $\mathcal{R}_{0}>1$ & $\mathscr{E}_{\mathbf{0}}$ is a saddle and $\mathscr{E}_{\mathbf{2}}$ is globally stable \\
\hline $\mathscr{E}_{\mathbf{i}}, i=0,1,2$ & $\tilde{\mathcal{R}}_{\Delta}<\mathcal{R}_{0}<1$ & $\begin{array}{c}\text { Both } \mathscr{E}_{\mathbf{0}} \text { and } \mathscr{E}_{\mathbf{2}} \text { are locally stable and } \\
\mathscr{E}_{\mathbf{1}} \text { is a saddle. }\end{array}$ \\
\hline
\end{tabular}

Table 3: Global dynamics. $A_{0}^{*}=\frac{k_{1}}{k_{2}}, \mathcal{R}_{0}=\frac{A_{0}^{*}}{A_{f}^{*}}, \mathcal{R}_{A}=\frac{\beta A_{0}^{*}}{k_{1}+\gamma}, \mathcal{R}_{\Delta}=\frac{k_{1}}{\phi_{\max }}$, and $\tilde{\mathcal{R}}_{\Delta}=\max \left(\mathcal{R}_{A}, \mathcal{R}_{\Delta}\right) . A_{f}^{*}$ and $\phi_{\max }$ are given in (7) \& (11) respectively. 


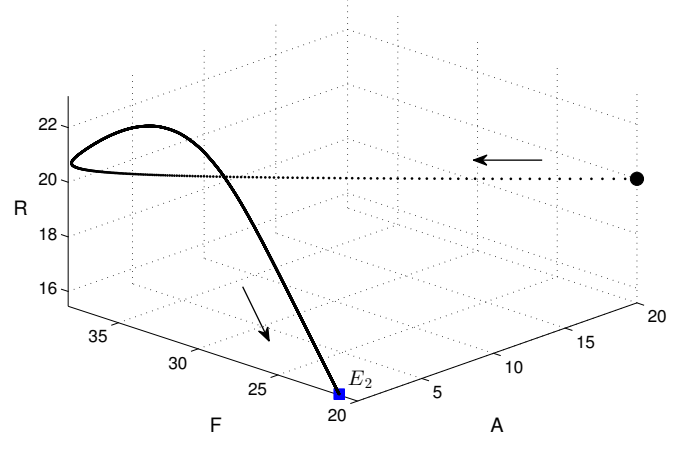

(a) One interior equilibrium $\left(\mathcal{R}_{0}>1\right)$

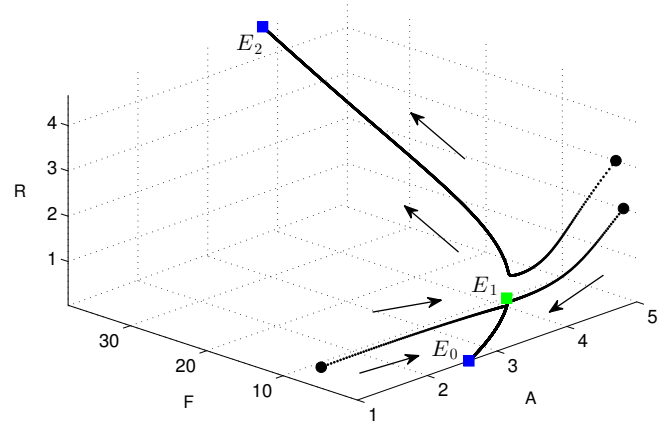

(b) Two interior equilibria $\left(\tilde{\mathcal{R}}_{\Delta}<\mathcal{R}_{0}<1\right)$

Figure 4: Phase space diagrams for model (4). Starting from initial conditions (filled circles), trajectories approach a unique globally stable foraging equilibrium $\left(\mathscr{E}_{\mathbf{2}}\right)\left(\right.$ a). Conversely, trajectories approach either the non-foraging equilibrium $\left(\mathscr{E}_{\mathbf{0}}\right)$ or the foraging equilibrium $\left(\mathscr{E}_{2}\right)$ which are both locally stable whenever $\tilde{\mathcal{R}}_{\Delta}<\mathcal{R}_{0}<1$ (b). Notice that the unstable equilibrium $\left(\mathscr{E}_{1}\right)$ comprises a separatrix that partitions the phase space into a region where foraging can persist.

Notes: Based on our analytical results in Theorem 3.1-3.2 and their corollary 3.1, we conclude that the foraging model (4) exhibits two kinds of global dynamics (see Fig. 3).

1. If $R_{c}<0$ (i.e., $\frac{\mathcal{R}_{0}}{\mathcal{R}_{A}}<1$ ), then model (4) undergoes a forward (transcritical) bifurcation as $\mathcal{R}_{0}$ increases past $\min \left\{1, \mathcal{R}_{A}\right\}$ characterized by the emergence of a globally stable foraging activity equilibrium $\mathscr{E}_{\mathbf{2}}$ (see Fig. 3(a)).

2. If $R_{c}>0$ (i.e., $\frac{\mathcal{R}_{0}}{\mathcal{R}_{A}}>1$ ), then model (4) undergoes a backward (subcritical) bifurcation as $\mathcal{R}_{0}$ decreases past one and a saddle-node bifurcation at $\tilde{\mathcal{R}}_{\Delta}=\max \left\{\mathcal{R}_{A}, \mathcal{R}_{\Delta}\right\}$. The backward bifurcation creates a region of bi-stability for the non-foraging equilibrium $\mathscr{E}_{\mathbf{0}}$ and the foraging equilibrium $\mathscr{E}_{\mathbf{2}}$ within $\tilde{\mathcal{R}}_{\Delta}<\mathcal{R}_{0}<1$ (see Fig. 3(b)).

As summarized in Corollary 3.1, our analysis indicates that the forager generation number $\left(\mathcal{R}_{0}\right)$ and the secondary threshold $\left(\tilde{\mathcal{R}}_{\Delta}\right)$ play essential roles in the dynamics of model (4) through their impacts on forager recruitment and availability (see Remark 3.1 \& Fig. 3). Thus, our results go significantly further than previous models (e.g., [24, 29, 30]) because they describe biologically meaningful conditions under which colonies can reach stable levels of activity, using parameters that reflect both colony-specific and ecological variables (Table 3).

Starting from low levels of activity, colonies can quickly reach high foraging levels if the average number of recruits per returning forager becomes greater than one $\left(\mathcal{R}_{0}>1\right)$. However, subsequent reductions of $\mathcal{R}_{0}$ below one may not necessarily cause the colony to suspend activity as long as $\mathcal{R}_{0}>\tilde{\mathcal{R}}_{\Delta}$ (Fig. $3(\mathrm{~b})$ ). From a biological standpoint, the degree to which colonies can absorb reductions of $\mathcal{R}_{0}$ (e.g., due to environmental perturbations of forager return rates) can be envisaged to reflect resilience or foraging robustness. To explore this idea, it is important to identify which parameters have greater sway on foraging dynamics, particularly through their impacts on $\mathcal{R}_{0}$ and the size of the foraging basin of attraction in model (4) (i.e., the set of initial conditions for which the colony reaches stable foraging levels). This is our focus in the next subsection.

\subsection{Connecting Bistability with Foraging Robustness}

As mentioned in Corollary 3.1, model (4) is bistable whenever $\tilde{\mathcal{R}}_{\Delta}<\mathcal{R}_{0}<1$. In this case, a twodimensional separatrix manifold partitions the basins of attraction (or attraction regions) of the non-foraging $\left(\mathscr{E}_{\mathbf{0}}\right)$ and foraging $\left(\mathscr{E}_{\mathbf{2}}\right)$ equilibria in $\mathbb{R}_{+}^{3}$ (Fig. 4). Biologically, this means that there exists a critical foraging size: $\mathscr{D}=(\tilde{A}, \tilde{F}, \tilde{R})$ below which the colony ultimately ceases foraging, and above which foraging activity 


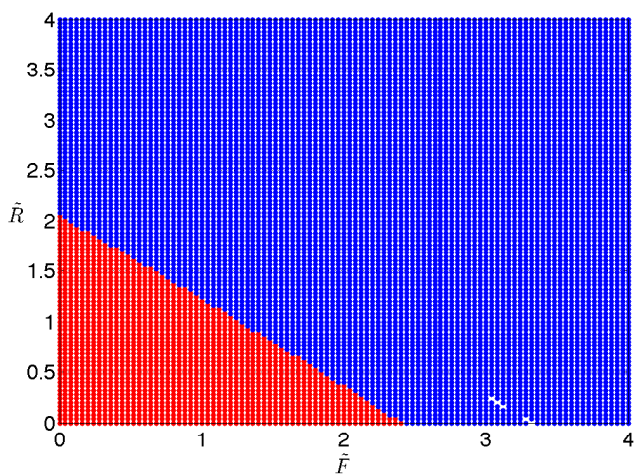

(a) $\xi=0.256$

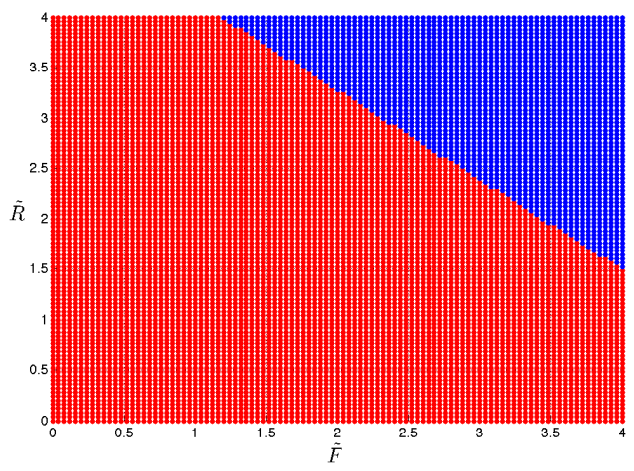

(b) $\xi=0.143$


(b) $\xi=0.143$. The critical number of returning foragers necessary to sustain activity scales nonlinearly with the number of active foragers for any fixed number of available foragers $(\tilde{A})$. However, no such relationship exists between available and active foragers (not shown). Finally, decreasing $\xi$ by less than $50 \%$ more than doubles the attraction region for $\mathscr{E}_{0}$. Other parameters: $k_{1}=.5, k_{2}=.2, \gamma=.03, \alpha=.04, d_{f}=2 d_{r}=.005$.

can persist (Fig. 5). There is a natural relationship between $\mathcal{R}_{0}, \tilde{\mathcal{R}}_{\Delta}$, and the attraction regions for $\mathscr{E}_{0}$ and $\mathscr{E}_{\mathbf{2}}$. Suppose that $\mathcal{R}_{\Delta}=\mathcal{R}_{\Delta}>\mathcal{R}_{A}$, then we can define the quantity:

$$
\xi=\mathcal{R}_{0}-\tilde{\mathcal{R}}_{\Delta}=\mathcal{R}_{0} \cdot\left(1-\frac{\tilde{\mathcal{R}}_{\Delta}}{\mathcal{R}_{0}}\right)=\frac{k_{1}}{k_{2} A^{*}} \cdot\left(1-\frac{4\left(\beta A^{*}-\gamma\right) k_{2} A^{*}}{\left(\beta A^{*}-\gamma+k_{1}\right)^{2}}\right) .
$$

Simulations show that the magnitude of $\xi$ correlates with the foraging basin of attraction (i.e., the attraction region of $\mathscr{E}_{2}$, Fig. 5). However, to better understand and quantify how small changes in one (or more) model parameters will affect the size of this region, we will use sensitivity analysis [39]. More specifically, we employ the normalized forward sensitivity index:

$$
\Gamma_{p}^{\mathbf{u}}:=\lim _{\delta p \rightarrow 0} \frac{\left(\frac{\delta \mathbf{u}}{\mathbf{u}}\right)}{\left(\frac{\delta p}{p}\right)}=\frac{p}{\mathbf{u}} \frac{\partial \mathbf{u}}{\partial p} \quad \mathbf{u} \neq 0
$$

where $\mathbf{u}$ is a differentiable output variable of interest and $p$ is a nominal input parameter [39]. The normalized sensitivity index $\left(\Gamma_{p}^{\mathbf{u}}\right)$ effectively estimates the expected percent change of a focal quantity (e.g., $\left.\mathcal{R}_{0}\right)$ given a unit percentage change (i.e., $\pm 1 \%$ ) of one of its component parameters (e.g., $\gamma$ ). Moreover, because parameters can be classified into those that likely depend on colony-specific properties (i.e., $k_{1}, k_{2}, \& \beta$ ), and those that reflect environmental conditions (e.g., $\alpha, \gamma$, etc.), the sensitivity index $\left(\Gamma_{p}^{\mathbf{u}}\right)$ can provide some intuition about which components colonies might regulate to improve short-term robustness to foraging perturbations.

From equations (18) and (19) it is straightforward to derive expressions defining $\Gamma_{p}^{\mathcal{R}_{0}}$ and $\Gamma_{p}^{\tilde{\mathcal{R}}_{\Delta}}$ as a function of their parameters. Table 4 lists the predicted indices based on the default parameter values compiled in Table (1). In general, parameters that increase $\mathcal{R}_{0}$ tend to decrease $\tilde{\mathcal{R}}_{\Delta}$ (although not always proportionally). For instance, the sensitivity index: $\Gamma_{\gamma}^{\mathcal{R}_{0}}=-1.48$ indicates that a $1 \%$ increase in the forager turnover rate should decrease $\mathcal{R}_{0}$ by $1.48 \%$ and vice versa (Table 4 ).

Based on the results, we predict that changes in both the worker arrival rate at the vestibule $\left(k_{1}\right)$ and the effective interaction rate $(\beta)$ should have the largest positive impacts on $\xi$, and thus the size of the attraction region of $\mathscr{E}_{\mathbf{2}}$. On the other hand, changes in the forager turnover rate $(\gamma)$ should have the largest negative 


\begin{tabular}{cccc}
\hline & \multicolumn{3}{c}{ Sensitivity Index $\left(\Gamma_{p}^{u}\right)$} \\
\cline { 2 - 4 } Parameter & $\mathcal{R}_{0}$ & $\tilde{\mathcal{R}_{\Delta}}$ & $\xi$ \\
\hline$k_{1}$ & +0.99 & -0.97 & +1.97 \\
$\beta$ & +0.99 & 0 & +0.99 \\
$\alpha$ & +0.11 & -0.603 & +0.713 \\
$d_{r}$ & -0.07 & +0.42 & -0.49 \\
$d_{f}$ & -0.11 & +0.603 & -0.713 \\
$k_{2}$ & -1.00 & 0 & -1.00 \\
$\gamma$ & -0.92 & +0.56 & -1.48 \\
\hline
\end{tabular}

Table 4: Normalized sensitivity indices $\left(\Gamma_{p}^{u}\right)$ of $\mathcal{R}_{0}, \tilde{\mathcal{R}}_{\Delta}$ in order of impact on $\xi=\mathcal{R}_{0}-\tilde{\mathcal{R}}_{\Delta}$. Sensitivity indices (dimensionless) quantify the expected percent change (positive or negative) in each quantity given a $1 \%$ perturbation of a component parameter. Results show that changes in the worker arrival rate $\left(k_{1}\right)$ and the forager turnover rate $(\gamma)$ should yield respectively the largest net positive and negative change in $\xi$.

impact on $\xi$, followed by the retirement rate $\left(k_{2}\right)$. Intuitively, we expect that colonies with larger values of $\xi$ will have a larger region of bistability below $\mathcal{R}_{0}<1$. This may reflect greater resilience to perturbations that further reduce $\mathcal{R}_{0}$ (e.g., due to increased predation on the foraging trail [35]). Estimating $\xi$ may therefore be useful for evaluating inter-colony differences in responsiveness and foraging robustness under comparable environmental conditions as has been observed in experiments [4, 31, 24].

Finally, our results here suggest that colonies should favor investments in regulating $k_{1}, k_{2}$, and $\beta$ as these are the only parameters that yield equal or greater returns in modulating $\xi$ (Table 4 ). Moreover, as our bifurcation analysis indicates, colony-specific parameters like the worker arrival rate at the vestibule $\left(k_{1}\right)$ play a critical role in the overall dynamics of activity through their impacts on forager availability (Fig. $3)$. For instance, if $k_{1}$ is small such that $\frac{\mathcal{R}_{0}}{\mathcal{R}_{A}}<1$, then foraging is highly vulnerable to perturbations that decrease $\mathcal{R}_{0}$ below one (Fig. 3(a)). On the other hand, if $k_{1}$ is large such that $\frac{\mathcal{R}_{0}}{\mathcal{R}_{A}}>1$, then foraging may persist despite perturbations that decrease $\mathcal{R}_{0}$ below one (Fig. 3(b)). These observations highlights the regulatory importance of the colony-specific parameters in our model.

\section{Numerical Simulations}

Previous experiments suggest that colonies of the seed harvester ant $P$. barbatus may use successful forager return rates to regulate overall foraging activity via worker interactions $[17,19,30]$. Our theoretical results predict that $\mathcal{R}_{0}$, and $\tilde{\mathcal{R}}_{\Delta}$ will have a significant influence on a colony's foraging activity. In this section, we will begin with an empirical validation of model (4) by performing a model fit to experimental data. In addition, we explore a dynamic sensitivity analysis of (4) to identify parameters (in different bifurcation regions) that drive model dynamics, and quantify their effects at both equilibrium (un-disturbed) and perturbed foraging states.

\subsection{Model Validation}

To examine whether our model simulates realistic responses to perturbations, we replicate in silico the experimental perturbation described in [17]. In multiple observations over a 3-day period, returning foragers of four mature P. barbatus (colonies: N1, N4, N5, \& N7 ) were artificially prevented from entering the nest for either 3 or 10 minutes during periods of high foraging activity. Throughout the trial, the numbers of available foragers in the vestibule along with the numbers of outgoing and returning foragers were recorded. In most instances, the number of outgoing foragers declined in response to the removals and recovered to varying levels of activity once returning foragers were allowed to return to the nest.

To estimate model parameters we used the averaged time-series for each colony (see Table 5). Along with fixed experimental parameters (e.g., times of removal) we generated best-fit response curves corresponding 

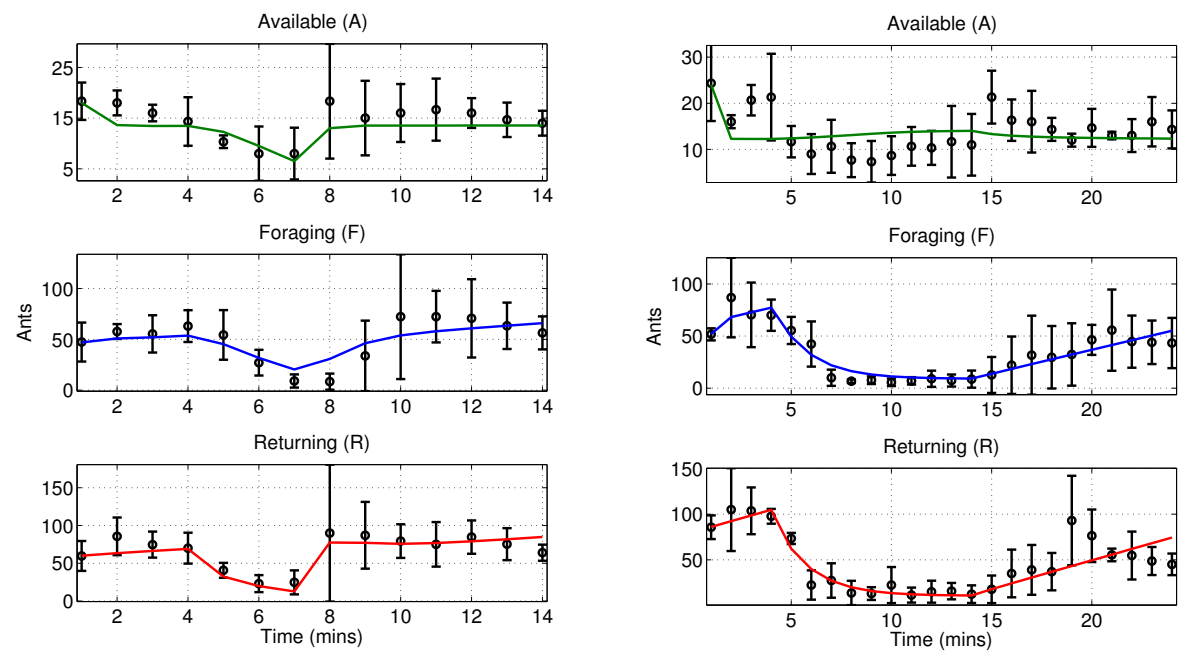

(a) 3 minute removal

(b) 10 minute removal

Figure 6: Simulations of forager removal perturbation experiment described in Pinter-Wollman et al. [17]. Model (4) was fitted (bold lines) to averaged time-series (circles) of available (top), outgoing (middle), and returning (bottom) foragers for colony N7. Error-bars show the variances between 3 trials reported in Pinter-Wollman et al. [17]. Removals began at $t=4$ and lasted either 3 minutes (left panel) or 10 minutes (right panel). Parameter estimates are given in Table 5

\begin{tabular}{ccccc}
\hline & \multicolumn{4}{c}{ Estimated Values (Colony N7) } \\
\cline { 2 - 5 } Parameter & 3-min removal & SD & 10-min removal & SD \\
\hline$\alpha$ & .6354 & .3930 & 8.251 & 15.325 \\
$\beta$ & .0386 & .0236 & .5003 & .8878 \\
$\gamma$ & .4568 & .2973 & 6.028 & 11.493 \\
$k_{1}$ & 11.067 & 12.783 & 10.763 & 6.532 \\
$k_{2}$ & 34.642 & 57.712 & 1.729 & 6.056 \\
\hline
\end{tabular}

Table 5: Estimated parameters (scaled in minutes) and one-standard deviations for colony N7. Estimates were generated using the Nonlinear grey-box modeling toolbox provided in MATLAB ${ }^{\circledR}$. Death rates were fixed prior and after the perturbation $\left(d_{f}=d_{r}=0, t<4 \& t>14\right)$ and estimated for returning foragers for 3-minute removals: (a) $d_{r}=1.01101 \pm 0.39937, t \in[4,7]$; and 10-minute removals (b) $d_{r}=1.01085 \pm 0.278261, t \in[4,14]$. Sampling intervals are same as in Table 1.

to observations for both 3 and 10 minute removals. Because the quality of fits did not vary extensively among colonies, we show results for one representative colony (N7) (Fig. 6).

The parameter estimates of $\mathcal{R}_{0}$ and $\tilde{\mathcal{R}}_{\Delta}$ after the perturbation indicate that colony activity was in the bistable foraging region during the 3 minute removals $\left(\mathcal{R}_{0}=.03, \tilde{\mathcal{R}}_{\Delta}=.0011\right)$ and 10 minute removal $\left(\mathcal{R}_{0}=.52, \tilde{\mathcal{R}}_{\Delta}=.18\right)$. We found similar values for colonies $\mathrm{N} 1$ and $\mathrm{N} 4$ (not shown). These results are consistent with empirical observations noting that harvester ant colonies will suppress and sometimes suspend foraging altogether if there are persistent declines in the forager return rate [4].

The low estimates of $\tilde{\mathcal{R}}_{\Delta}$, particularly in the 3 minute removals, do not suggest that foraging can be recovered without recruitment. In the context of our model, the low values reflect the increased potential of colonies to recover from short-term versus long-term interruptions. We stress that the ability of colonies to maintain their activity, in the bistable foraging region (i.e., $\tilde{\mathcal{R}}_{\Delta}<\mathcal{R}_{0}<1$ ) depends critically on the distribution of the forager workforce between the available, active, and returning states (Fig. 4). In terms of biological significance, these estimates likely reflect the fact that harvester ant colonies are not very sensitive to foraging interruptions once they have reached high stable levels of activity [4, 21, 40]. 
Finally, we acknowledge some model limitations may have affected the goodness of fit. Most obviously, our model does not capture any biological stochasticity. Thus, we cannot comment extensively on the strength of our estimates of $\mathcal{R}_{0}$ and $\tilde{\mathcal{R}}_{\Delta}$ to predict the recovery dynamics after the perturbation when the number of foragers becomes very small. Furthermore, we do not account for spatial constraints of vestibule size and structure which will influence the baseline numbers of available foragers, interaction patterns inside the vestibule, and any delays affecting the re-availability of returning foragers once inside the nest. Indeed, a summary analysis of the Pinter-Wollman et al. [17] activity data revealed a lagged correlation between the numbers of ants in the vestibule and number of returning foragers (Pearson's product-moment correlation, $\mathrm{t}=1.9592, \mathrm{df}=13, \mathrm{p}=0.07189)$. Such delays should be accounted for in future models.

\subsection{Simulated Perturbation 85 Dynamic Sensitivity Analysis}

It is often the case in non-linear systems that the relative importance of one or more parameters on the observed dynamics might change under different conditions. Our results so far suggest that changes in four key parameters: $k_{1}, \beta, k_{2} \& \gamma$, should greatly influence the behavior of model (4) because of their predicted effects on $\mathcal{R}_{0}$ and $\tilde{\mathcal{R}}_{\Delta}$ (see Table 4 ). In the context of a foraging colony, the relative importance of these parameters may change depending on whether activity is close to or far away from equilibrium (e.g., due to a foraging perturbation). In the Appendix, we explore this question by performing a dynamic sensitivity analysis of model (4) during a perturbation. Our results predict varying patterns of sensitivity for each parameter, during equilibrium and non-equilibrium periods (Fig. 7(a)-7(d)). In general, these effects were most pronounced at the height of the perturbations, again highlighting the potential regulatory importance of these parameters. However, we caveat that the predicted sensitivity patterns depend on our model formalism, and may diverge from predictions of an analogous discrete system when populations are small.

\section{Discussion}

We have developed a simple model describing the interaction-based recruitment and regulation of collective foraging in harvester ant colonies $[4,17,19,21]$. Our main result is that depending on model parameters, foraging activity can either persist at high levels or may advance toward one of two coexisting attractors corresponding to an intermediate or non-foraging state. The dynamical predictions of model (4) detailed in Theorem 3.1-3.2, Remark 3.1, and Corollary 3.1 can be summarized as follows:

1. Foraging is persistent whenever the expected number of recruits per successful returning forager, over the typical duration of a foraging trip, is larger than one (i.e., $\mathcal{R}_{0}>1$ ).

2. If $\mathcal{R}_{0}$ is less than one, foraging may still be sustained if $\tilde{\mathcal{R}}_{\Delta}<\mathcal{R}_{0}$ which results in two possibilities: either (i) $\mathcal{R}_{0}$ is larger than the ratio of the worker arrival rate into the vestibule and the maximum rate of change of non-retiring foragers (i.e., $\mathcal{R}_{0}>\mathcal{R}_{\Delta}=\frac{k_{1}}{\phi_{\max }}>\mathcal{R}_{A}=\frac{\beta A_{0}^{*}}{\left(k_{1}+\gamma\right)}$ ) or (ii) $\mathcal{R}_{0}$ is larger than the ratio of the expected recruitment rate for a successful returning forager and the average rate of increase of available foragers in the vestibule when the colony is near its non-foraging equilibrium (i.e., $\left.\mathcal{R}_{0}>\mathcal{R}_{A}=\frac{\beta A_{0}^{*}}{\left(k_{1}+\gamma\right)}>\mathcal{R}_{\Delta}=\frac{k_{1}}{\phi_{\max }}\right)$.

Previous modeling studies have assumed that returning foragers have a constant effect on the rate of forager recruitment without identifying any underlying causal parameters [24, 29, 30]. Here, we identified the forager generation number $\left(\mathcal{R}_{0}\right)$ as a threshold quantity. $\mathcal{R}_{0}$ is influenced by a combination of internal and external variables including the baseline number of available foragers, effective forager interaction rates inside the vestibule, and the average duration of a foraging trip (13). Moreover, because the baseline recruitment rate (i.e., $\beta A_{0}^{*}$ ) is determined by colony-specific activity levels which determine the rates at which workers arrive to $\left(k_{1}\right)$, retire from $\left(k_{2}\right)$, and interact in $(\beta)$ the vestibule, we suggest that $\mathcal{R}_{0}$ may be regulated by the colony relative to external conditions. Indeed, ants alter encounter rates, as a function of food availability $[17]$. 


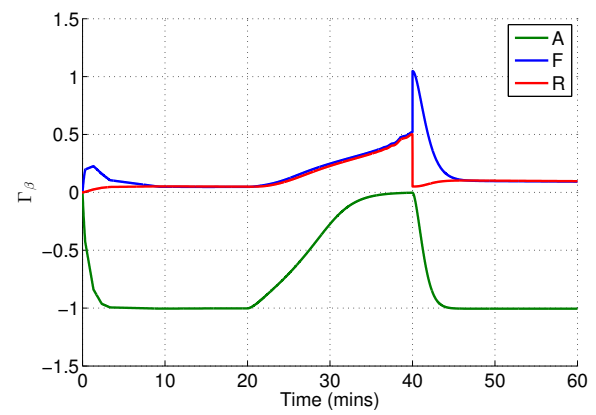

(a) $\beta$

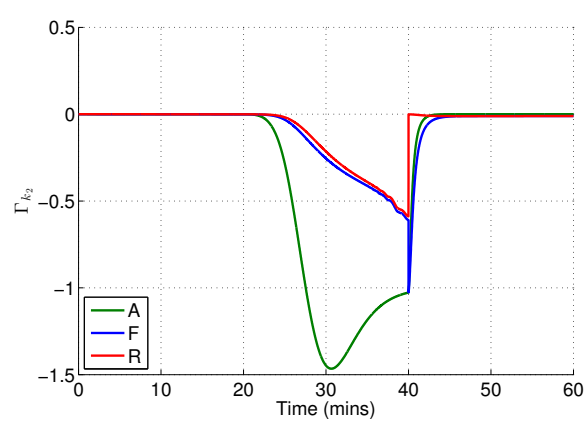

(c) $k_{2}$

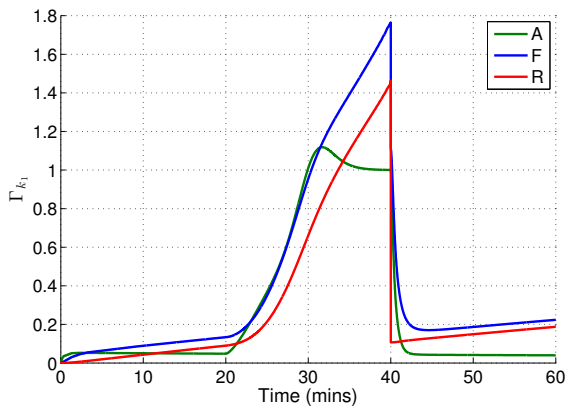

(b) $k_{1}$

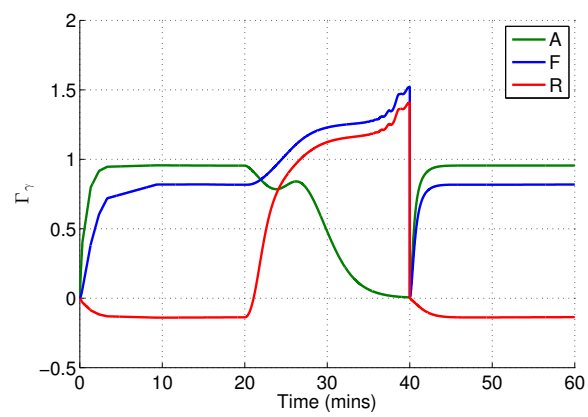

(d) $\gamma$

Figure 7: Dynamic model sensitivity w.r.t. select parameters during simulated perturbations: (a) Effective interaction rate, $\beta$; (b) Worker arrival rate, $k_{1}$; (c) Retirement rate, $k_{2}$; (d) Forager turnover rate, $\gamma$. The model is initialized at equilibrium in the bistable foraging region $\tilde{\mathcal{R}}_{\Delta}<\mathcal{R}_{0}<1$, where $\mathcal{R}_{0}=.19, \tilde{\mathcal{R}}_{\Delta}=.01$. A perturbation is introduced between $20<t<40$ by increasing returning forager death rates. Sensitivity indices (y-axis, dimensionless) describe the expected percentage change in the numbers of available foragers (green), activated foragers (blue) or returning foragers (red) given a $1 \%$ increase in the focal parameter.

Our model also makes testable predictions about the expected number of available foragers in the vestibule as a function of recruitment (13): (i) colonies in the persistent activity region (i.e., $\mathcal{R}_{0}>1$ ) are likely to have large numbers of available foragers during periods of low forager returns such as when the weather becomes warmer and drier, and relatively smaller numbers as forager return rates increase. (ii) colonies in the bistable foraging region (i.e., $\tilde{\mathcal{R}}_{\Delta}<\mathcal{R}_{0}<1$ ) are likely to have large numbers of available foragers during periods of high forager returns such as when food availability is high, and relatively smaller numbers as forager return rates decline (see Table 3 \& Fig. 8). Interestingly, these observations suggest that colonies should show varied patterns of recovery from a sudden perturbation, such as predation by a horned lizard, depending on whether they are in the persistent or bistable foraging region. Because the rate at which foraging resumes after a perturbation depends primarily on the numbers of available foragers in the vestibule [17], our results suggest that colonies in the bistable region may recover faster from small, short-term reductions in forager return rates. Conversely, colonies in the persistent region may recover faster from large, long-term reductions in forager return rates. Although we did not have sufficient data to test this prediction, the insight highlights the advantage of our modeling approach over previous attempts (e.g., [29]) in that we can better connect and understand the dynamics of forager availability and recruitment with varied patterns of foraging activity.

Our analysis and simulations confirm that the size of the bistable foraging region (i.e., $\xi=\mathcal{R}_{0}-\tilde{\mathcal{R}}_{\Delta}$ ) correlates with the size of the foraging basin of attraction in model (4) (Fig. 3 \& Fig. 5). Thus, we suggest that $\xi$ could theoretically serve as an indicator of foraging robustness, predicting that colonies with larger values of $\xi$ should be more resilient to declines in forager return rates and foraging interruptions compared 
to colonies with smaller vales of $\xi$. Along similar lines, sensitivity analysis of $\mathcal{R}_{0}$ suggests that it will most likely be impacted by colony-specific properties that determine average worker arrival rate $\left(k_{1}\right)$ and forager retirement rate $\left(k_{2}\right)$, as well as the forager interaction rate $(\beta)$ and turnover rate $(\gamma)$ (see Table 4). These results suggest that modulating the movement of foragers from the inner nest to the vestibule (and viceversa) along with the management of interaction rates inside the vestibule are important components likely to have the greatest impact on the regulation of foraging activity. This prediction is consistent with the results of a recent study, which demonstrated that harvester ant colonies regulate their foraging activity at multiple timescales providing plasticity and robustness to short and long term foraging perturbations [17].

An important biological insight of our results is that an interaction-based forager recruitment strategy shares many of the regulatory strengths of leader-based recruitment (e.g., tandem running), and selforganizing chemical recruitment (e.g., pheromone trails). Although tandem running conveys more direct information about foraging conditions, which can allow the colony to flexibly respond to short-term changes in environmental profitability, it critically lacks the mass-recruitment properties of self-reinforcing pheromone trails [11]. On the other hand, the strong reinforcing feedbacks that associate trail quality with forager usage and pheromone strength can often weaken the colony's ability to flexibly track changes in resource quality [16]. Moreover, the fidelity of pheromone-based trail networks is often limited by volatility and degradation under variable environmental conditions outside the control of the colony. An interaction-based recruitment strategy circumvents these limitations because it is self-organizing (in that every returning forager is a potential recruiter), and because it can be more adaptively controlled by the colony. This could be achieved, for instance, if available foragers alter their interaction rates inside the vestibule in response to fluctuations in forager return rates as seen in Pinter-Wollman et al. [17]. Interaction rates may change by altering walking patterns, for example an increased path tortuosity will reduce interaction rate as shown both theoretically [2] and empirically [41] for this ant species.

Our results additionally suggest that the dynamics of forager availability in the vestibule may play a particularly important role in sustaining activity when forager return rates are low (Table 4 \& Fig 7). The vestibule must be populated sparsely, not densely, to keep forager activation rates high. Because an individual worker's retirement decision is made based on her perception of local forager densities inside the vestibule, colonies might differ in their level of responsiveness to forager return rates simply based on worker response thresholds. Indeed, recent works by [17, 24] have shown that colonies can vary substantially in the differences between per-capita interaction rates (within the vestibule) during periods of high versus low forager return, and in the effects of humidity on forager activation rates.

In conclusion, our results support the evidence that a simple interaction-based recruitment strategy can provide a robust regulatory system for managing collective foraging in ant colonies [17, 21]. Furthermore, our model provides useful insights into how internal and external variables can impact foraging dynamics, including identifying potential sources of inter-colony variability in activity patterns [24]. Although our model is based on the foraging ecology of harvester ant colonies, our framework can be generalized to analogous systems that rely on local worker interactions (e.g., nest construction in social wasps [43], quorum-sensing in rock ants, [44], etc).

The current model, however, does not include some potentially important regulatory components that have been hypothesized $[17,24]$. Two such examples include potential regulatory feedbacks between forager return rates and worker arrival rates, as well as the effects of spatial constraints on interaction patterns inside the vestibule. A simple modification of our model, can link worker arrival rates to the number of available foragers, and employ a saturating forager recruitment term. These complexities will be addressed in our future work. 


\section{Proofs}

Proof of Theorem 3.1

Proof. According to the formulation of the foraging model (4), the following holds for $(A, F, R) \in \mathbb{R}_{+}^{3}$ :

$$
\begin{aligned}
& \left.A^{\prime}\right|_{A=0}=k_{1}+\gamma R \geq k_{1} \\
& \left.F^{\prime}\right|_{F=0}=\beta A R \geq 0 \\
& \left.R^{\prime}\right|_{R=0}=\alpha F \geq 0 .
\end{aligned}
$$

Thus, applying the results of Theorem A.4, p.423 in Thieme [47], we can conclude that the foraging model (4) is positively invariant in $\mathbb{R}_{+}^{3}$.

Let $N=A+F+R$, then we have

$$
N^{\prime}=A^{\prime}+F^{\prime}+R^{\prime}=k_{1}-\frac{k_{2} A}{1+R}-d_{f} F-d_{r} R .
$$

Thus, we have the following inequalities based on the property of the positivity invariance:

$$
k_{1}-\max \left\{k_{2}, d_{f}, d_{r}\right\} N \leq k_{1}-k_{2} A-d_{f} F-d_{r} R \leq N^{\prime} \leq \frac{k_{1}(1+R)-k_{2} A-d_{f} F-d_{r} R(1+R)}{1+R} .
$$

This indicates the following two cases:

\section{Bounded below:}

$$
N^{\prime} \geq k_{1}-\max \left\{k_{2}, d_{f}, d_{r}\right\} N \Rightarrow \liminf _{t \rightarrow \infty} N(t) \geq \frac{k_{1}}{\max \left\{k_{2}, d_{f}, d_{r}\right\}} .
$$

\section{Bounded above:}

$$
\begin{aligned}
N^{\prime} & \leq \frac{k_{1}(1+R)-k_{2} A-d_{f} F-d_{r} R(1+R)}{1+R}=\frac{k_{1}(1+R)-d_{r} R^{2}-k_{2} A-d_{f} F-d_{r} R}{1+R} \\
& \leq \frac{k_{1}(1+R)-d_{r} R^{2}-\min \left\{k_{2}, d_{f}, d_{r}\right\} N}{1+R}=\frac{-d_{r}\left(R-\frac{k_{1}}{2 d_{r}}\right)^{2}+\frac{k_{1}^{2}}{4 d_{r}}+k_{1}-\min \left\{k_{2}, d_{f}, d_{r}\right\} N}{1+R} \\
& \leq \frac{\frac{k_{1}^{2}}{4 d_{r}}+k_{1}-\min \left\{k_{2}, d_{f}, d_{r}\right\} N}{1+R} .
\end{aligned}
$$

This indicates that

$$
\limsup _{t \rightarrow \infty} N(t) \leq \frac{k_{1}+\frac{k_{1}^{2}}{4 d_{r}}}{\min \left\{k_{2}, d_{f}, d_{r}\right\}} .
$$

Therefore, we can conclude that every trajectory starting in $\mathbb{R}_{+}^{3}$ is attracted to the following compact set

$$
C=\left\{(A, F, R) \in \mathbb{R}_{+}^{3}: \frac{k_{1}}{\max \left\{k_{2}, d_{f}, d_{r}\right\}} \leq A+F+R \leq \frac{k_{1}+\frac{k_{1}^{2}}{4 d_{r}}}{\min \left\{k_{2}, d_{f}, d_{r}\right\}}\right\}
$$

which also implies that the foraging dynamics of model (4) can be restricted to the compact set $C$.

Let $M=\frac{k_{1}+\frac{k_{1}^{2}}{4 d_{r}}}{\min \left\{k_{2}, d_{f}, d_{r}\right\}}$. Because model (4) is bounded by $M$, the number of returning ants $R$ is also bounded by $M$. This implies that for any $\epsilon>0$, there exists time $T$ large enough, such that we have

$$
A^{\prime}=k_{1}-\beta A R+\gamma R-k_{2} \frac{A}{1+R} \geq k_{1}-\beta(M+\epsilon) A-k_{2} A \geq k_{1}-\left(\beta(M+\epsilon)+k_{2}\right) A, \text { for all } t>T .
$$


This indicates that

$$
\liminf _{t \rightarrow \infty} A(t) \geq \frac{k_{1}}{\beta M+k_{2}} .
$$

Therefore, we can conclude that $A$ is persistent in $\mathbb{R}_{+}^{3}$.

Proof of Theorem 3.2

Proof. Because the non-foraging equilibrium $\mathscr{E}_{\mathbf{0}}=\left(A_{0}^{*}, 0,0\right)=\left(\frac{k_{1}}{k_{2}}, 0,0\right)$ always exists, we focus on sufficient conditions lead to the existence of the foraging equilibrium $\mathscr{E}_{\mathbf{i}}=\left(A_{f}^{*}, F_{i}^{*}, R_{i}^{*}\right)=\left(A_{f}^{*}, \frac{\gamma+d_{r}}{\alpha} R_{i}^{*}, R_{i}^{*}\right), i=$ 1,2 where $A_{f}^{*}=\frac{\left(\alpha+d_{f}\right)\left(\gamma+d_{r}\right)}{\alpha \beta}$ and $R_{i}^{*}$ are roots of the equation $\phi\left(R^{*}\right)=k_{2} A_{f}^{*}$ with

$$
\phi(R)=\left[k_{1}-\left(\beta A_{f}^{*}-\gamma\right) R\right](1+R) .
$$

Therefore, the existence of $\mathscr{E}_{\mathrm{i}}$ is determined by the positive intercept(s) of the quadratic function $\phi(R)$ and the horizontal line $k_{2} A_{f}^{*}$, which can be classified into the following two cases depending on the sign of the critical point $R_{c}=\frac{\gamma-\beta A_{f}^{*}+k_{1}}{2\left(\beta A_{f}^{*}-\gamma\right)}$ of $\phi(R)$ (see Fig. 2(b)):

1. If $R_{c}<0$ (see Fig. $2(\mathrm{a})$ ), then we have

$$
R_{c}=\frac{\gamma-\beta A_{f}^{*}+k_{1}}{2\left(\beta A_{f}^{*}-\gamma\right)}<0 \Leftrightarrow A_{f}^{*}>\frac{k_{1}+\gamma}{\beta} \Leftrightarrow k_{1}<d_{r}+\frac{d_{f}\left(\gamma+d_{r}\right)}{\alpha} \Leftrightarrow \mathcal{R}_{0}=\frac{k_{1}}{k_{2} A_{f}^{*}}<\frac{k_{1} \beta}{k_{2}\left(k_{1}+\gamma\right)} .
$$

In this case, the foraging dynamics can have $\mathscr{E}_{\mathbf{0}}$ or $\mathscr{E}_{\mathbf{i}}, i=0,2$ depending on the ratio of $\frac{\phi(0)}{k_{2} A_{f}^{*}}=\frac{k_{1}}{k_{2} A_{f}^{*}}$ :

(a) If $\frac{\phi(0)}{k_{2} A_{f}^{*}}=\frac{k_{1}}{k_{2} A_{f}^{*}}<1$ (i.e., $\mathcal{R}_{0}<1$, see the purple horizontal line in Fig. 2(a)), then either there is no intercept of the null clines or the intercepts of $\phi(R)$ and the horizontal line $k_{2} A_{f}^{*}$ are located in the black region (i.e., negative values). In this scenario, the foraging model (4) only has the non-foraging equilibrium $\mathscr{E}_{0}$.

(b) If $\frac{\phi(0)}{k_{2} A_{f}^{*}}=\frac{k_{1}}{k_{2} A_{f}^{*}}>1$ (i.e., $\mathcal{R}_{0}>1$, see the cyan horizontal line in Fig. 2(a)), then there is a unique foraging equilibrium $\mathscr{E}_{\mathbf{2}}$. Thus, in this scenario, the foraging model (4) has the non-foraging equilibrium $\mathscr{E}_{0}$ and the foraging equilibrium $\mathscr{E}_{2}$.

2. If $R_{c}>0$ (see Fig. 2(b)), then we have

$$
R_{c}=\frac{\gamma-\beta A_{f}^{*}+k_{1}}{2\left(\beta A_{f}^{*}-\gamma\right)}<0 \Leftrightarrow A_{f}^{*}<\frac{k_{1}+\gamma}{\beta} \Leftrightarrow k_{1}>d_{r}+\frac{d_{f}\left(\gamma+d_{r}\right)}{\alpha} \Leftrightarrow \mathcal{R}_{0}=\frac{k_{1}}{k_{2} A_{f}^{*}}>\frac{k_{1} \beta}{k_{2}\left(k_{1}+\gamma\right)} .
$$

In this case, the foraging dynamics can have $\mathscr{E}_{\mathbf{0}}$ or $\mathscr{E}_{\mathbf{i}}, i=0,2$ or $\mathscr{E}_{\mathbf{i}}, i=0,1,2$ depending on the ratio of $\frac{\phi(0)}{k_{2} A_{f}^{*}}=\frac{k_{1}}{k_{2} A_{f}^{*}}$ and $\frac{\phi_{\max }}{k_{2} A_{f}^{*}}$ :

(a) If $k_{2} A_{f}^{*}>\phi_{\max } \geq \phi(0)=k_{1}$, we have

$$
k_{2} A_{f}^{*}>\phi_{\max } \geq \phi(0)=k_{1} \Leftrightarrow 0<\mathcal{R}_{0}=\frac{k_{1}}{k_{2} A_{f}^{*}}<\mathcal{R}_{\Delta}=\frac{k_{1}}{\phi_{\max }} .
$$

In this case, the horizontal line $k_{2} A_{f}^{*}$ (see the purple horizontal line in Fig. 2(b)) is above the quadratic equation $\phi(R)$, i.e., there is no foraging equilibrium. Thus, in this scenario, the foraging model (4) has only the non-foraging equilibrium $\mathscr{E}_{0}$. 
(b) If $k_{1}<k_{2} A_{f}^{*}<\phi_{\max }$ (see the dark green horizontal line in Fig. 2(b)), then we have the following equalities:

$$
k_{1}<k_{2} A_{f}^{*}<\phi_{\max } \Leftrightarrow 0<\mathcal{R}_{\Delta}=\frac{k_{1}}{\phi_{\max }}<\frac{k_{1}}{k_{2} A_{f}^{*}}=\mathcal{R}_{0}<1<\frac{k_{2} A_{f}^{*}}{\phi_{\max }} .
$$

In this scenario, the foraging model (4) has the non-foraging equilibrium $\mathscr{E}_{\mathbf{0}}$ and two foraging equilibria $\mathscr{E}_{\mathbf{i}}, i=1,2$.

(c) If $\frac{\phi(0)}{k_{2} A_{f}^{*}}=\frac{k_{1}}{k_{2} A_{f}^{*}}>1$ (i.e., $\mathcal{R}_{0}>1$, see the cyan horizontal line in Fig. $2(\mathrm{~b})$ ), then there is a unique foraging equilibrium $\mathscr{E}_{\mathbf{2}}$. Thus, in this scenario, the foraging model (4) has the non-foraging equilibrium $\mathscr{E}_{0}$ and the foraging equilibrium $\mathscr{E}_{2}$.

Now we focus on the local stability of the non-foraging equilibrium $\mathscr{E}_{\mathbf{0}}$ and two foraging equilibria $\mathscr{E}_{\mathbf{i}}, i=1,2$ when they exist. The local stability of $\mathscr{E}_{\mathbf{0}}$ is determined by the eigenvalues $\lambda_{i}, i=1,2,3$ of the Jacobian matrix associated with the foraging model (4)

$$
\left.J\right|_{\mathscr{E}_{\mathbf{O}}}:=\left[\begin{array}{ccc}
-k_{2} & 0 & -\frac{\beta k_{1}}{k_{2}}+k_{1}+\gamma \\
0 & -\left(\alpha+d_{f}\right) & \frac{\beta k_{1}}{k_{2}} \\
0 & \alpha & -\left(\gamma+d_{r}\right)
\end{array}\right]
$$

where

$\lambda_{1}=-k_{2}, \lambda_{2}+\lambda_{3}=-\left(\alpha+d_{f}+d_{r}+\gamma\right)<0$, and $\lambda_{2} \lambda_{3}=\frac{-\alpha \beta k_{1}}{k_{2}}+\left(\gamma+d_{r}\right)\left(\alpha+d_{f}\right)=\left(\gamma+d_{r}\right)\left(\alpha+d_{f}\right)\left[1-\mathcal{R}_{0}\right]$.

This indicates that if $\mathcal{R}_{0}<1$, then $\lambda_{i}<0, i=1,2,3$; while if $\mathcal{R}_{0}>1$, then $\lambda_{i}>0,2,3$. Therefore, $\mathscr{E}_{0}$ is locally asymptotically stable if $\mathcal{R}_{0}<1$ and it is a saddle if $\mathcal{R}_{0}>1$.

The Jacobian matrix evaluated at $\mathscr{E}_{i}, i=1,2$ can be represented as follows:

$$
\left.J\right|_{\mathscr{E}_{i}}:=\left[\begin{array}{ccc}
-\beta R_{i}^{*}-\frac{k_{2}}{1+R_{i}^{*}} & 0 & -\beta A_{f}^{*}+\gamma+\frac{k_{2} A_{f}^{*}}{\left(1+R_{i}^{*}\right)^{2}} \\
\beta R_{i}^{*} & -\left(\alpha+d_{f}\right) & \beta A_{f}^{*} \\
0 & \alpha & -\left(\gamma+d_{r}\right)
\end{array}\right]
$$

whose eigenvalues satisfy the characteristic polynomial:

$$
\rho(\lambda)=\lambda^{3}+c_{2} \lambda^{2}+c_{1} \lambda+c_{0}=0
$$

with

$$
\begin{aligned}
& c_{2}=\beta R_{i}^{*}+\left(\alpha+d_{f}+d_{r}+\gamma\right)+\frac{k_{2}}{1+R_{i}^{*}}>0 \\
& c_{1}=\left(\alpha+d_{f}+d_{r}+\gamma\right)\left[\beta R_{i}^{*}+\frac{k_{2}}{1+R_{i}^{*}}\right]>0 \\
& c_{0}=\beta R_{i}^{*}\left[\left(\gamma+d_{r}\right) d_{f}+\alpha d_{r}\right]-\frac{k_{2} R_{i}^{*}\left(\gamma+d_{r}\right)\left(\alpha+d_{f}\right)}{\left(1+R_{i}^{*}\right)^{2}}
\end{aligned}
$$

According to the Routh-Hurwitz criteria [45], we conclude that the foraging equilibrium $\mathscr{E}_{\mathbf{i}}$ is locally asymptotically stable if and only if $c_{1} c_{2}>c_{0}>0$. According to (21), we have

1. $c_{1} c_{2}>\beta R_{i}^{*}\left(\alpha+d_{f}+d_{r}+\gamma\right)^{2}$ indicates that

$$
c_{1} c_{2}-c_{0}>\beta R_{i}^{*}\left(\alpha+d_{f}+d_{r}+\gamma\right)^{2}-\beta R_{i}^{*}\left[\left(\gamma+d_{r}\right) d_{f}+\alpha d_{r}\right]>0 .
$$

Because $c_{1} c_{2}>c_{0} \Leftrightarrow c_{1} c_{2}-c_{0}>0$, thus, we can conclude $c_{1} c_{2}>c_{0}$ always holds for both $R_{i}^{*}, i=1,2$. 
2. The following equivalent relationships holds

$$
\begin{array}{rlrl}
c_{0}>0 & \Leftrightarrow & \beta\left(1+R_{i}^{*}\right)^{2}\left[\left(\gamma+d_{r}\right) d_{f}+\alpha d_{r}\right]-k_{2}\left(\gamma+d_{r}\right)\left(\alpha+d_{f}\right)>0 \\
& \Leftrightarrow & & \left(1+R_{i}^{*}\right)^{2}>\frac{k_{2}\left(\gamma+d_{r}\right)\left(\alpha+d_{f}\right)}{\beta\left[\left(\gamma+d_{r}\right) d_{f}+\alpha d_{r}\right]}=\frac{k_{2} A_{f}^{*}}{\beta A_{f}^{*}-\gamma}
\end{array}
$$

Notice that $0<R_{1}^{*}<R_{2}^{*}$ are roots of $\phi(R)=k_{2} A_{f}^{*}$, thus we have

$$
\phi\left(R_{i}^{*}\right)=\left[k_{1}-\left(\beta A_{f}^{*}-\gamma\right) R_{i}^{*}\right]\left(1+R_{i}^{*}\right)=k_{2} A_{f}^{*} \Leftrightarrow\left[\frac{k_{1}}{\beta A_{f}^{*}-\gamma}-R_{i}^{*}\right]\left(1+R_{i}^{*}\right)=\frac{k_{2} A_{f}^{*}}{\beta A_{f}^{*}-\gamma} .
$$

This indicates that

$$
\begin{aligned}
c_{0}>0 & \Leftrightarrow\left(1+R_{i}^{*}\right)^{2}>\left[\frac{k_{1}}{\beta A_{f}^{*}-\gamma}-R_{i}^{*}\right]\left(1+R_{i}^{*}\right) \\
& \Leftrightarrow 1+R_{i}^{*}>\frac{k_{1}}{\beta A_{f}^{*}-\gamma}-R_{i}^{*} \\
& \Leftrightarrow \quad R_{i}^{*}>\frac{k_{1}}{2\left(\beta A_{f}^{*}-\gamma\right)}-\frac{1}{2}
\end{aligned}
$$

Recall that

$$
\begin{aligned}
& R_{1}^{*}=\frac{k_{1}}{2\left(\beta A_{f}^{*}-\gamma\right)}-\frac{1}{2}-\sqrt{\left(\frac{k_{1}}{2\left(\beta A_{f}^{*}-\gamma\right)}-\frac{1}{2}\right)^{2}+\frac{k_{1}-k_{2} A_{f}^{*}}{\beta A_{f}^{*}-\gamma}} \\
& R_{2}^{*}=\frac{k_{1}}{2\left(\beta A_{f}^{*}-\gamma\right)}-\frac{1}{2}+\sqrt{\left(\frac{k_{1}}{2\left(\beta A_{f}^{*}-\gamma\right)}-\frac{1}{2}\right)^{2}+\frac{k_{1}-k_{2} A_{f}^{*}}{\beta A_{f}^{*}-\gamma}}
\end{aligned}
$$

Then we have

$$
\begin{aligned}
& R_{1}^{*}<\frac{k_{1}}{2\left(\beta A_{f}^{*}-\gamma\right)}-\frac{1}{2} \Rightarrow c_{0}<0 \\
& R_{2}^{*}>\frac{k_{1}}{2\left(\beta A_{f}^{*}-\gamma\right)}-\frac{1}{2} \Rightarrow c_{0}>0
\end{aligned}
$$

The discussion above implies that if the foraging equilibrium $\mathscr{E}_{\mathbf{i}}$ exists, then $\mathscr{E}_{\mathbf{1}}$ is always a saddle and $\mathscr{E}_{\mathbf{2}}$ is always locally asymptotically stable.

Proof of Corollary 3.1

Proof. According to Theorem 3.1, every trajectory of the foraging model (4) attracts to a compact set $C$ defined in (5). Thus we can restrict the dynamics of model (4) in this compact set $C$. Based on the proof of Theorem 3.2, we have the following three cases:

1. If $\mathcal{R}_{0}<\min \left\{1, \frac{k_{1} \beta}{k_{2}\left(k_{1}+\gamma\right)}\right\}$ or $\frac{k_{1} \beta}{k_{2}\left(k_{1}+\gamma\right)}<\mathcal{R}_{0}<\mathcal{R}_{\Delta}$, then the foraging model (4) has only the non-foraging equilibrium $\mathscr{E}_{\mathbf{0}}=\left(\frac{k_{1}}{k_{2}}, 0,0\right)$ which is locally asymptotically stable. model (4) enters a compact set $C$ and has a unique locally stable equilibrium $\mathscr{E}_{\mathbf{0}}$. Thus, $\mathscr{E}_{\mathbf{O}}$ is globally stable by applying the results of Poincare-Bendixson trichotomy in three dimensional systems [46].

2. If $\mathcal{R}_{0}>1$, then the foraging model (4) has the non-foraging equilibrium $\mathscr{E}_{\mathbf{0}}$ and the foraging equilibrium $\mathscr{E}_{\mathbf{2}}$ where $\mathscr{E}_{\mathbf{0}}$ is a saddle and $\mathscr{E}_{\mathbf{2}}$ is locally asymptotically stable. Therefore, by applying the results of Poincare-Bendixson trichotomy in three dimensional systems [46], we can conclude that every trajectory starting with strict positive initial condition converges to $\mathscr{E}_{2}$, i.e., and $\mathscr{E}_{2}$ is globally stable. 


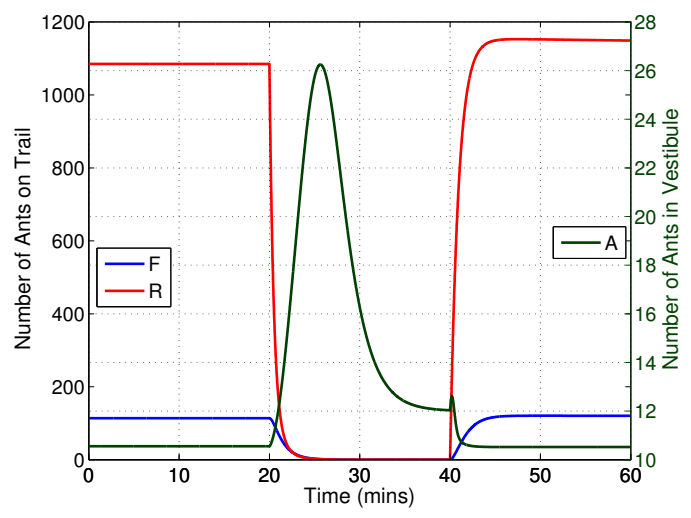

(a) Persistent foraging region

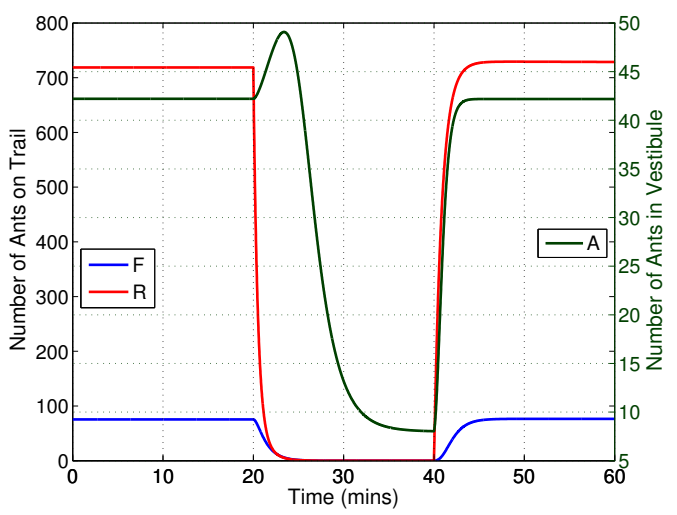

(b) Bistable foraging region

Figure 8: Dynamics of model (4) under perturbation. The model is initialized at equilibrium with parameters satisfying (a) Persistent region: $\mathcal{R}_{0}=1.19$, (b) Bistability region: $\mathcal{R}_{0}=.19>.01=\tilde{\mathcal{R}}_{\Delta}$. A perturbation is introduced at $t=20$ by increasing returning forager death rate, $d_{r}$ by $2000 \%$ (red). The number of available foragers (green) increases initially as forager activations declines (blue).

3. If $\max \left\{\frac{k_{1} \beta}{k_{2}\left(k_{1}+\gamma\right)}, \mathcal{R}_{\Delta}\right\}<\mathcal{R}_{0}<1$, then the foraging model (4) has the non-foraging equilibrium $\mathscr{E}_{\mathbf{0}}$ and two foraging equilibria $\mathscr{E}_{\mathbf{i}}, i=1,2$ where both $\mathscr{E}_{\mathbf{0}}$ and $\mathscr{E}_{\mathbf{2}}$ are locally asymptotically stable. This implies that the foraging model (4) has bistability, i.e., depending on the initial condition, the trajectory may converge to the non-foraging equilibrium $\mathscr{E}_{\mathbf{0}}$ or the foraging equilibrium $\mathscr{E}_{\mathbf{2}}$.

\section{Appendix on Dynamic Sensitivity Analysis}

For model (4), we are particularly interested in quantifying how possible changes in $k_{1}, \beta, k_{2} \& \gamma$ will influence the number of foragers in each state over time because of their predicted effects on $\mathcal{R}_{0}$ and $\tilde{\mathcal{R}}_{\Delta}$ (see Table 4). Mathematically, dynamic sensitivity analysis is done by solving a set of adjoining forward sensitivity equations obtained by taking the partial derivatives of (4) with respect to its component parameters (see [39] for a review). We employed the ODE15s SENS_SYS extension in MATLAB written by V.M. Garcia Molla, which calculates and integrates the sensitivity equations. The output is a time-series of model sensitivities that we normalized using equation (19).

We initialized system (4) at the interior foraging equilibrium with parameters in the: (i) persistent activity region $\left(\mathcal{R}_{0}>1\right.$ ), and (ii) bistablity region $\left(\tilde{\mathcal{R}}_{\Delta}<\mathcal{R}_{0}<1\right.$ ) (see Fig. 3(b)). We then introduce a constant perturbation by increasing the returning forager death rate similar to the experimental removals described in [17] (i.e., $d_{r}=0.05, t<20 ; d_{r}=1, t \in[20,40]$ ).

Figures $8(\mathrm{a})$ and $8(\mathrm{~b})$ show the effects of forager removal on model dynamics. As the perturbation increases beyond $t=20$, the number of available workers in the vestibule rises initially due to insufficient interactions with returning foragers. However, as we continue to prevent foragers from returning, forager retirement rates ultimately overtake arrival rates at the vestibule, reducing the pool of available foragers. This happens at around $t=25$. Finally, at $t=40$, all numbers increase as foragers are allowed to return to the nest, and the system tends back towards equilibrium.

In general, the model tends to equilibrate worker availability faster than empirically observed. This difference between model and experiments is likely due to the model assumptions that there is no direct recruitment from the inner nest to the vestibule. Future work adding dynamics to the rate of worker arrival from the inner nest $\left(k_{1}\right)$ might uncover further foraging regulation mechanisms. 
There are major differences between the system's response during and after the perturbation period, depending on the parameter region. In the persistent foraging region, the average number of available foragers in the absence of returning foragers is relatively higher than it is when there is activity. Conversely, in the bistable region, the average numbers of available foragers in the absence of returning foragers is much lower than it is when there is activity (Fig. 8). At first glance, this result is counter-intuitive because one might expect that colonies with persistently high foraging levels should have larger numbers of available foragers. However, recall that the total expected rate of forager availability in the persistent region is always less than in the bistable region (i.e., $\phi_{2}>\phi_{1}$, see Fig. 2). This is not only because $\mathcal{R}_{0}$ is an increasing function of the effective interaction rate, $\beta$ ) (see Table 4), but also because forager availability is a non-decreasing function of $\mathcal{R}_{0}$ (14). Based on these observations, we can infer that the equilibrium number of available foragers should be relatively lower in the persistent region due to the increased rate of forager recruitment.

Figures 7(a)-7(d) show the corresponding model sensitivities for select parameters as a function of time. The normalized sensitivity indices (y-axes) shows the expected percentage change in the numbers of available foragers (green), activated foragers (blue) or returning foragers (red) given a $1 \%$ increase in the focal parameter (see equation (19)). Our results predict varying patterns of sensitivity for each parameter, during equilibrium and non-equilibrium periods. Because our model is deterministic and continuous we cannot discuss stochasticity which would appear in a discrete model and might affect the sensitivity of the parameters in small populations. However, because the populations we model are large (thousands of ants) our continuous model provides an plausible approximation of a discrete system.

1. Figure 7 (a) shows that at equilibrium (i.e., $t<20, t>40$ ), increasing the effective interaction rate $(\beta)$ by a percent should have a negative impact on available foragers $(\approx 1 \%$ reduction $)$ and a small but positive impact on both active and returning foragers. However, as the perturbation unfolds (i.e., $20 \leq t \leq 40$ ), its expected impact on both active and returning foragers increases steadily and peaks near the end of the perturbation period as the numbers of available foragers begin to increase. As discussed previously, the initial increase in the number of ants in the vestibule results from a reduction in forager activation rates. These observations suggest that modulating interaction rates in the vestibule should be particularly important to the colony especially during a perturbation when there might be fewer numbers of returning and available foragers.

2. Figures $7(\mathrm{~b})-7(\mathrm{c})$ show respectively the impacts of increasing the worker arrival rate $\left(k_{1}\right)$ and the forager retirement rate $\left(k_{2}\right)$ on the model's dynamics. Increasing worker arrivals always has a positive impact on all compartments, especially during the perturbation (Fig. 7(b)). However, the impact of $k_{1}$ is generally greater on both active and returning foragers particularly when the model approaches equilibrium. Conversely, the effect of increasing the forager retirement rate at foraging equilibrium is almost negligible in all compartments (Fig. 7(c)) However, as the perturbation unfolds, all three compartments show increasing negative sensitivity to $k_{2}$ with its largest impact on available foragers $(\approx 1.4 \%$ reduction $)$. Together, these observations suggest from the colony's perspective that the joint modulation of worker arrival and forager retirement rates will be a key component in maintaining foraging activity especially during a perturbation. We would expect colonies to work towards increasing worker arrival rates during periods of high returns, and decreasing the forager retirement rate during periods of low returns in order to stabilize their forager recruitment rates.

3. Figure $7(\mathrm{~d})$ shows the effects of the forager turnover rate $(\gamma)$ on the model dynamics. At equilibrium, increasing $\gamma$ by a percent will have a positive, but diminished impact on the numbers of available and active foragers (i.e., $<1 \%$ ). However, as the perturbation unfolds, its predicted impact is largest and positive for both active and returning foragers. This observation is intuitive: when returning foragers are in decline, increasing $\gamma$ increases forager availability in the vestibule. This in turn will have an effect on the numbers of active foragers through its positive impact on the forager recruitment rate. Although we assume that $\gamma$ is constant, the reality is that it will likely subject to some important feedbacks from other dynamical variables not captured in our model such as the current work demand at the nest which may alter forager behavior once inside, and influence how quickly they become re- 
available for recruitment. These types of feedbacks will impact the sensitivity patterns observed during a perturbation.

\section{Acknowledgements}

O.U's research is supported by the Simon A. Levin Mathematical, Computational and modeling Sciences Center (SAL-MCMSC) in part through a Graduate Assistance in Areas of National Need Fellowship (DOEGAANN) grant. Y.K's research is partially supported by NSF-DMS (Award Number 1313312), Simons Collaboration Grants for Mathematicians (208902) and the research scholarship from School of Letters and Sciences. NPW was funded by the NIH P50 San Diego Center for Systems Biology (SDCSB) grant \#GM085764.

\section{References}

[1] Detrain, C., Deneubourg, J. L., \& Pasteels, J. M. (1999). Decision-making in foraging by social insects. In Information processing in social insects (pp. 331-354). Birkhäuser Basel.

[2] Adler, F. R., \& Gordon, D. M. (1992). Information collection and spread by networks of patrolling ants. American Naturalist, 373-400.

[3] Beshers, S. N., \& Fewell, J. H. (2001). Models of division of labor in social insects. Annual review of entomology, 46(1), 413-440.

[4] Gordon, D. M. (2002). The regulation of foraging activity in red harvester ant colonies. The American Naturalist, 159(5), 509-518.

[5] Fewell, J. H., \& Winston, M. L. (1992). Colony state and regulation of pollen foraging in the honey bee, Apis mellifera L. Behavioral Ecology and Sociobiology, 30(6), 387-393.

[6] Bonabeau, E., Theraulaz, G., Deneubourg, J. L., Aron, S., \& Camazine, S. (1997). Self-organization in social insects. Trends in Ecology $\&$ Evolution, 12(5), 188-193.

[7] Dornhaus, A., \& Chittka, L. (2004). Information flow and regulation of foraging activity in bumble bees (Bombus spp.). Apidologie, 35(2), 183-192.

[8] Höldobler, B., \& Wilson, E. O. (1990). The ants. Harvard University Press, Cambridge.

[9] Franks, N. R., \& Richardson, T. (2006). Teaching in tandem-running ants. Nature, 439(7073), 153-153.

[10] Fernandez, A. A., \& Deneubourg, J. L. (2011). On following behaviour as a mechanism for collective movement. Journal of theoretical biology, 284(1), 7-15.

[11] Traniello, J. F. (1989). Foraging strategies of ants. Annual review of entomology, 34(1), 191-210.

[12] Collignon, B., Deneubourg, J. L., \& Detrain, C. (2012). Leader-based and self-organized communication: Modelling group-mass recruitment in ants. Journal of theoretical biology, 313, 79-86.

[13] Sumpter, D., \& Pratt, S. (2003). A modelling framework for understanding social insect foraging. Behavioral Ecology and Sociobiology, 53(3), 131-144

[14] Deneubourg, J. L., Goss, S., Franks, N., \& Pasteels, J. M. (1989). The blind leading the blind: modeling chemically mediated army ant raid patterns. Journal of insect behavior, 2(5), 719-725.

[15] Tabone, M., Ermentrout, B., \& Doiron, B. (2010). Balancing organization and flexibility in foraging dynamics. Journal of theoretical biology, 266(3), 391-400. 
[16] Dussutour, A., \& Nicolis, S. C. (2013). Flexibility in collective decision-making by ant colonies: Tracking food across space and time. Chaos, Solitons 83 Fractals, 50, 32-38.

[17] Pinter-Wollman, N., Bala, A., Merrell, A., Queirolo, J., Stumpe, M. C., Holmes, S., \& Gordon, D. M. (2013). Harvester ants use interactions to regulate forager activation and availability. Animal behaviour, 30(1), e11.

[18] Gordon, D. M., Holmes, S., \& Nacu, S. (2008). The short-term regulation of foraging in harvester ants. Behavioral Ecology, 19(1), 217-222.

[19] Greene, M. J., \& Gordon, D. M. (2007). Interaction rate informs harvester ant task decisions. Behavioral Ecology, 18(2), 451-455.

[20] Gordon, D. M. (2010). Ant encounters: interaction networks and colony behavior. Princeton University Press.

[21] Greene, M. J., Pinter-Wollman, N., \& Gordon, D. M. (2013). Interactions with combined chemical cues inform harvester ant foragers' decisions to leave the nest in search of food. PloS one, 8(1), e52219.

[22] Beverly, B. D., McLendon, H., Nacu, S., Holmes, S., \& Gordon, D. M. (2009). How site fidelity leads to individual differences in the foraging activity of harvester ants. Behavioral Ecology, 20(3), 633-638.

[23] Lighton, J. R., \& Feener Jr, D. H. (1989). Water-loss rate and cuticular permeability in foragers of the desert ant Pogonomyrmex rugosus. Physiological Zoology, 1232-1256.

[24] Gordon, D. M., Dektar, K. N., \& Pinter-Wollman, N. (2013). Harvester ant colony variation in foraging activity and response to humidity. PloS one, 8(5), e63363.

[25] Gordon, D. M. (1998). Task-related differences in the cuticular hydrocarbon composition of harvester ants, Pogonomyrmex barbatus. Journal of Chemical Ecology, 24(12), 2021-2037.

[26] Wagner, D., Brown, M. J., Broun, P., Cuevas, W., Moses, L. E., Chao, D. L., \& Gordon, D. M. (1998). Task-related differences in the cuticular hydrocarbon composition of harvester ants, Pogonomyrmex barbatus. Journal of Chemical Ecology, 24(12), 2021-2037.

[27] Pacala, S. W., Gordon, D. M., \& Godfray, H. C. J. (1996). Effects of social group size on information transfer and task allocation. Evolutionary Ecology, 10(2), 127-165.

[28] Nicolis, S. C., Theraulaz, G., \& Deneubourg, J. L. (2005). The effect of aggregates on interaction rate in ant colonies. Animal behaviour, 69(3), 535-540.

[29] Prabhakar, B., Dektar, K. N., \& Gordon, D. M. (2012). The regulation of ant colony foraging activity without spatial information. PLoS computational biology, 8(8), e1002670.

[30] Schafer, R. J., Holmes, S., \& Gordon, D. M. (2006). Forager activation and food availability in harvester ants. Animal Behaviour, 71(4), 815-822.

[31] Gordon, D. M., Guetz, A., Greene, M. J., \& Holmes, S. (2011). Colony variation in the collective regulation of foraging by harvester ants. Behavioral Ecology, 22(2), 429-435.

[32] Gordon, D. M. (1991). Behavioral flexibility and the foraging ecology of seed-eating ants. American Naturalist, 379-411.

[33] Gordon, D. M., \& Kulig, A. W. (1996). Founding, foraging, and fighting: colony size and the spatial distribution of harvester ant nests. Ecology, 2393-2409.

[34] Gordon, D. M. (1996). The organization of work in social insect colonies. Nature, 380(6570), 121-124. 
[35] Munger, J. C. (1984). Long-term yield from harvester ant colonies: implications for horned lizard foraging strategy. Ecology, 1077-1086.

[36] Pol, R. G., Lopez de Casenave, J., \& Pirk, G. I. (2011). Influence of temporal fluctuations in seed abundance on the foraging behaviour of harvester ants (Pogonomyrmex spp.) in the central Monte desert, Argentina. Austral Ecology, 36(3), 320-328.

[37] Johnson, R. A. (1991). Learning, memory, and foraging efficiency in two species of desert seed-harvester ants. Ecology, 1408-1419.

[38] De Vita, J. (1979). Mechanisms of interference and foraging among colonies of the harvester ant Pogonomyrmex californicus in the Mojave Desert. Ecology, 729-737.

[39] Arriola, L., \& Hyman, J. M. (2009). Sensitivity analysis for uncertainty quantification in mathematical models. In Mathematical and Statistical Estimation Approaches in Epidemiology (pp. 195-247). Springer Netherlands.

[40] Jandt, J. M., Bengston, S., Pinter-Wollman, N., Pruitt, J. N., Raine, N. E., Dornhaus, A., \& Sih, A. (2014). Behavioural syndromes and social insects: personality at multiple levels. Biological Reviews, 89(1), 48-67.

[41] Pinter-Wollman, N., Wollman, R., Guetz, A., Holmes, S., \& Gordon, D. M. (2011). The effect of individual variation on the structure and function of interaction networks in harvester ants. Journal of The Royal Society Interface, 8(64), 1562-1573.

[42] Oldroyd, B. P., \& Fewell, J. H. (2007). Genetic diversity promotes homeostasis in insect colonies. Trends in Ecology \& Evolution, 22(8), 408-413.

[43] Jeanne, R. L. (1986). The organization of work in Polybia occidentalis: costs and benefits of specialization in a social wasp. Behavioral Ecology and Sociobiology, 19(5), 333-341.

[44] Pratt, S. C. (2005). Quorum sensing by encounter rates in the ant Temnothorax albipennis. Behavioral Ecology, 16(2), 488-496.

[45] Brauer, F., \& Castillo-Chavez, C. (2012). Mathematical models in population biology and epidemiology. Springer.

[46] Thieme, H. R. (1992). Convergence results and a Poincare-Bendixson trichotomy for asymptotically autonomous differential equations. Journal of mathematical biology, 30(7), 755-763.

[47] Thieme, H. R. (2003). Mathematics in population biology. Princeton University Press.

[48] Smith, H. L., \& Thieme, H. R. (2011). Dynamical systems and population persistence (Vol. 118). American Mathematical Soc. 